Microfluidics for Production of Particles : Mechanism, Methodology, and Applications

\title{
Liu, Zehua
}

2020-03-05

Liu , Z , Fontana , F , Python, A , Hirvonen , J T \& Santos , H A 2020 , ' Microfluidics for Production of Particles : Mechanism, Methodology, and Applications ', Small, vol. 16 , no. 9 , 1904673 . https://doi.org/10.1002/smll.201904673

http://hdl.handle.net/10138/321179

https://doi.org/10.1002/smll.201904673

unspecified

acceptedVersion

Downloaded from Helda, University of Helsinki institutional repository.

This is an electronic reprint of the original article.

This reprint may differ from the original in pagination and typographic detail.

Please cite the original version. 


\section{WILEY-VCH}

DOI: 10.1002/ ((please add manuscript number))

\section{Article type: Review}

Title Microfluidics for production of particles: mechanism, methodology and applications

Zehua Liu, Flavia Fontana, Andre Python, Jouni T. Hirvonen, and Hélder A. Santos*

Dr. Z. Liu, Dr. F. Fontana, Prof. J. T. Hirvonen, Prof. H. A. Santos

Drug Research Program, Division of Pharmaceutical Chemistry and Technology

Faculty of Pharmacy

University of Helsinki

FI-00014 Helsinki, Finland

E-mail: helder.santos@helsinki.fi; Tel.+358 294159661

Prof. H. A. Santos

Helsinki Institute of Life Science (HiLIFE)

University of Helsinki

FI-00014 Helsinki, Finland

Dr. A. Python

Nuffield Department of Medicine

Li Ka Shing Centre for Health Information and Discovery

Big Data Institute

University of Oxford 


\section{WILEY-VCH}

OX3 7LF, Oxford, United Kingdom

Keywords: microfluidics; nanoparticles; microparticles; drug delivery; biological analysis 


\title{
WILEY-VCH
}

\begin{abstract}
In the past two decades, microfluidics-based particle production has been widely applied for multiple biological usages. Comparing to conventional bulk methods, microfluidic-assisted particle production shows significant advantages, such as narrower particle size distribution, higher reproducibility, improved encapsulation efficiency and enhanced scaling-up potency. In this review, we provide an overview of the recent progress of the microfluidics technology for nano-, micro-particles or droplet fabrication, and their biological applications. For both nano-, micro-particles/droplets, we discuss the previously established mechanisms behind particle production via microfluidics and highlight some typical examples during the past five years. The emerging interdisciplinary technologies based on microfluidics that have produced microparticles or droplets for cellular analysis and artificial cells fabrication are summarized. The potential drawbacks and future perspectives are also briefly discussed.
\end{abstract}




\section{WILEY-VCH}

\section{Introduction}

Particles and droplets production is one of the most indispensable and fundamental process in biomedical engineering field, and have been widely applied in controlled drug delivery/release, ${ }^{[1]}$ vaccination, ${ }^{[2]}$ tissue engineering, ${ }^{[3]}$ biosensors and diagnostic devices, ${ }^{[4]}$ bio-imaging, ${ }^{[5]}$ cellular analysis and artificial cells fabrication. ${ }^{[6,7]}$ Based on the size scale, particles can be divided into nano- and micro-particles and droplets. Yet, a better clinical translation of such nano-, micro-particles is highly dependent on the reproducible and scalable synthesis and production methodology, which can be barely concurrently achieved with bulk methods, such as high pressure homogenization (HPH), sonication or static mixer. ${ }^{[8]}$ As such, a sophisticated alternative method should be further applied to better control the production of nano-, micro-particles.

In past decades, the concept "Lab-on-Chip" facilitated the evolving development of continuous and more precise systems for healthcare applications. ${ }^{[9]}$ As a fundamental regime, microfluidics - a technology characterized by the manipulating nanoliters scale of fluids in submillimeter channels - has become an innovative alternative approach to the bulk method in the biomedical field. ${ }^{[10]}$ It has been widely applied in 3D cell cultures, single cell analysis, cell/molecule isolation and purification, body fluidic stream simulation, organ-on-chip, nano-, micro-fibers production, and nano-, micro-particles production. ${ }^{[11]}$

Microfluidics assisted particles and/or droplets production has drawn increasing attentions due to its several advantages including high reproducibility, low batch-to-batch variation, better control over particle characters and easy to scale-up. We identified the most frequent keywords associated with scientific articles using a word extraction procedure applied on a large database. The procedure allowed us to identify the tendency of microfluidic application over the past 10 


\section{WILEY-VCH}

years. We investigated changes in the frequency and order of appearance of the most frequent keywords extracted between two time periods: (i) 2009-2013 and (ii) 2014-2019. We adapted the R code (scopusapi.R) from https://github.com/christopherBelter/scopusAPI to implement a query on the word "microfluidics", using Scopus Search application programming interface (API) in R software [quote R here]. For each time period, we kept a maximum of 2000 most relevant downloaded records. We further queried the API, using an advanced search string to extract the authors' keywords, and computed their frequency of appearance, and ranked the keywords according to their frequency. Figure 1 (left panel) shows two cloud plots that highlight words that appear more than 10 times, and the top 25 most frequent words are shown Figure 1 (right panel). These results suggest that, despite their observed decrease in frequency in the later period, cellular analysis and microdroplet-based single cell analysis remain the most prevailing applications for microfluidics. We also noticed frequency changes between the investigated periods. For example, the word "drug" (ranked $12^{\text {th }}$ in $2009-2013$ ) became $3^{\text {rd }}$ in 2014-2019, and the word "delivery" only appeared on the "2014-2019 billboard". Furthermore, ranking for the word "capillary" shifted from $11^{\text {th }}(2009-2013)$ to $8^{\text {th }}(2014-2019)$ position and the word "paperbased" solely appeared on the top 25 most frequent words in 2014-2019, which may suggest a recent tendency for microfluidic chip fabrication. In addition to that, there are several words that only appeared in the 2014-2019 cloud plot, such as "particle", "engineering", “imaging", "culture", "film”, and "phase”, indicating increasing interests in using microfluidics for particle fabrication and engineering.

In this review, we will briefly describe recent advances of microfluidic-produced particles and/or droplets for biomedical applications. Dependent on the size of the produced particles, the review will be divided into two parts: nanoparticles, which are mainly produced via single- 


\section{WILEY-VCH}

phase flow microfluidics; and micro-particles/droplets, which are fabricated via multi-phase flow microfluidics. We will separately describe the previously established mechanism behind the microfluidics-assisted nano-, micro-particles or droplets production to better illustrate the advantages of the current systems. Moreover, we will highlight some recent publications using microfluidics-based nano-, micro-particles for multiple biomedical applications. For nanoparticles, we will focus on their microfluidics-assisted production with identical core/shell structure. For micro-particles/droplets, we will mainly highlight microfluidics-produced microparticles/droplets for biological analysis or simulation, which are inspired by the evolving molecular biological concepts and techniques, such as single cell analysis and/or artificial cells. Also, we will discuss the potential drawbacks and limitations of the current techniques and methods. 


\section{WILEY-VCH}

a

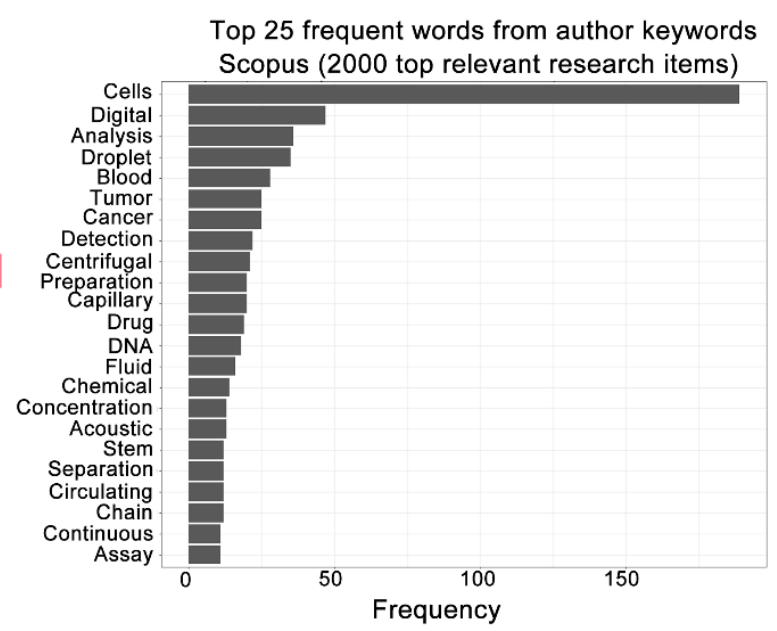

b
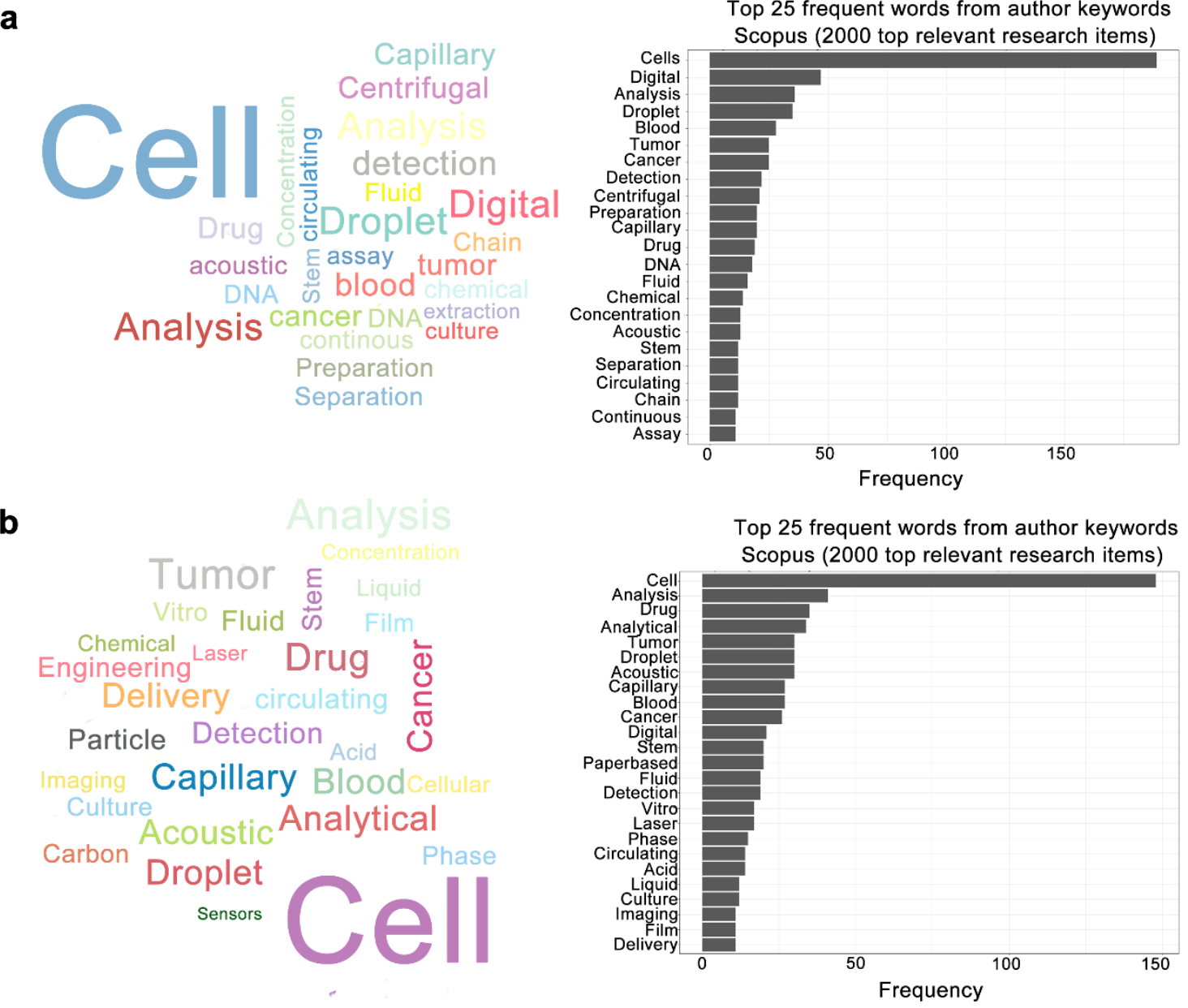

Figure 1. Visualisation of the most frequent words from author keywords of scientific papers using Scopus Search "microfluidics” over two time periods: (a) 2009-2013 and (b) 2014-2019. The left panel (cloud plot) highlights words that appear more than 10 times, and the right panel lists the top 25 frequent words screened from author keywords.

\section{Microfluidic production of nanoparticles}

\subsection{Advantages of microfluidics for synthesis of nanoparticles (NPs)}

Producing nanoparticles via the microfluidics method is not a new story. It has long been reported that the continuous synthesis of NPs by using microfluidics obtained better reproducibility and controllability compared to batch-type bulk synthesis methods. ${ }^{[12]}$ This is 


\section{WILEY-VCH}

mainly due to the mechanism of NPs formation and the unique fluid dynamics in the microfluidic systems.

In a typical NPs synthesis, the procedure is based on a bottom-up approach, where most of the theoretical work describing the formation process of the NPs are based on the classical nucleation theory. In 1950, LaMer et al. proposed the concept of burst nucleation, which described the formation of NPs as: (1) a rapid increase in the concentration of free monomers in solution, inducing a specific supersaturation level; (2) energy barrier for nucleation is overcame, leading to the burst nucleation; (3) burst nucleation rapidly decrease the supersaturation level, resulting in the termination of further nucleation; and (4) secondary growth occurs at the particle surfaces by diffusion facilitated monomer flux. ${ }^{[13]}$ Following work has enriched the theory by providing refined interpretation of both the growth and size narrowing processes. For example, Reiss deduced that the size focusing phenomenon from NPs formation was due to the diffusion induced growing of particles, which is solely dependent on the size of NPs where smaller NPs obtain a faster growth rate. ${ }^{[14]}$ Later on, Lifshitz, Slyozov and Wagner made a major advance by introducing the Ostwald ripening theory for interpreting the coarsening phenomenon of the particles (LSW theory). ${ }^{[14,15]}$ Ostwald ripening is the process by which small particles shrink, due to enhanced solubility arising from their high curvature, and larger particles grow. Ostwald ripening is commonly invoked to explain the particle aging, coarsening and stability, and readers may refer the following papers to have a further knowledge. ${ }^{[16]}$ So far, the LaMer's model and its following refinements are still the most commonly accepted models describing the NPs formation. ${ }^{[17,18,19]}$

The commonly accepted mathematical equation describing the nucleation is based on the Gibbs equation, which describes free energy of a nucleus $(\Delta G),{ }^{[18,19]}$ as defined in Eq. (1): 


$$
\Delta G=-\frac{4}{3} \pi r^{3}\left|\Delta G_{v}\right|+4 \pi r^{2} \gamma
$$

and, $\Delta \mathrm{G}_{\mathrm{v}}$ is further defined in Eq. (2):

$$
\Delta G_{v}=\frac{-k_{B} T \ln (S)}{v}
$$

where $\Delta \mathrm{G}_{\mathrm{v}}$ stands for free energy of the cluster, $\mathrm{r}$ stands for the spherical particle radius, $\gamma$ stands for the surface energy per unit area, $\mathrm{k}_{\mathrm{B}}$ stands for the Boltzmann's constant, $\mathrm{S}$ stands for the supersaturation level of the solution and $v$ stands for the molar volume of the monomer. When the radius of a nucleus reaches to a critical value $\left(r_{c}\right), \Delta G$ achieves the maximum value (energy barrier), thus the further growth of the cluster is favored $\left(\frac{\mathrm{d} \Delta G}{\mathrm{~d} r}<0\right.$, Figure 2a). This further gives the critical radius, as defined in Eq. (3):

$$
r_{c}=\frac{2 v \gamma}{k_{B} T \ln (S)}
$$

The nucleation rate $\left(\frac{\mathrm{d} N}{\mathrm{~d} t}\right)$ can be further expressed by the Arrhenius equation, as defined in Eq. (4):

$$
\frac{\mathrm{d} N}{\mathrm{~d} t}=A \exp \left[\frac{16 \pi r^{3} v^{2}}{3 k_{B}{ }^{3} T^{3}(\ln S)^{2}}\right]
$$

where, $\mathrm{A}$ is the pre-exponential factor and $\mathrm{T}$ is the temperature.

Nucleation critically impacts the quality of the formed NPs, as nucleation rate is positively correlated to the nuclei density, which will further result in smaller NPs and enhanced particle yield. ${ }^{[20-22]}$ Based on Eq. (3), controllable parameters (T, S and $\gamma$ ) can be varied to influence the nucleation rate. Kwon et al. plotted these three parameters, and suggested a change from $\mathrm{S}=2$ 


\section{WILEY-VCH}

to $S=4$ could cause an increase in the nucleation rate about $\sim 10^{70}$, and at room temperature $(\approx$ $300 \mathrm{~K}$ ), even slight temperature change could induce changes of several orders of magnitude in the nucleation rate (Figure $\mathbf{2 b - d}){ }^{[18]}$

In order to achieve homogeneous kinetics, the NPs nucleation and growth process should be preferably initiated in a homogenous solution, which means the mixing time of 2 solvents, $\mathrm{t}_{\mathrm{mix}}$, must be less than the time scale for the nucleation initiation, $t_{\text {ini. }}$ In a typical amphiphilic block polymer nucleation procedure, the relevant time scale for initial structure formation can be under $100 \mathrm{~ms}$ for molecules of $100000 \mathrm{~g} \mathrm{~mole}^{-1} \cdot{ }^{[23]}$ However, from a practical point of view, bulk methods usually fail to create a homogenous condition within the orders of milliseconds. ${ }^{[24]}$ As such, the local supersaturation level of the monomers within the solution may be vastly altered, and as a sequelae, results in a lower monodispersity and higher batch-to-batch variation. ${ }^{[22,25,26]}$

Microfluidics, a technology characterized by the engineered manipulation of fluids at the submillimeter scale, ${ }^{[27,28,29]}$ can sharply increase the surface area-to-volume ratio by several orders of magnitude, and therefore, allows for more efficient mass and heat transfer within the system. More importantly, microfluidic chips with specific modification and specific flow diameter can achieve the mixing time on the order of milliseconds, rendering a $t_{\text {mixing }}<t_{\text {ini }}$ regime, further yielding the particle formation within a homogeneous solution. The flowdominated mixing within the microfluidic channel provides better reproducibility and controllability compared to batch-type bulk mixing. Such feature makes the microfluidics a very convenient platform for the synthesis of NPs with better monodispersity and reduced batch-to-batch variation. ${ }^{[12]}$ 


\section{WILEY-VCH}
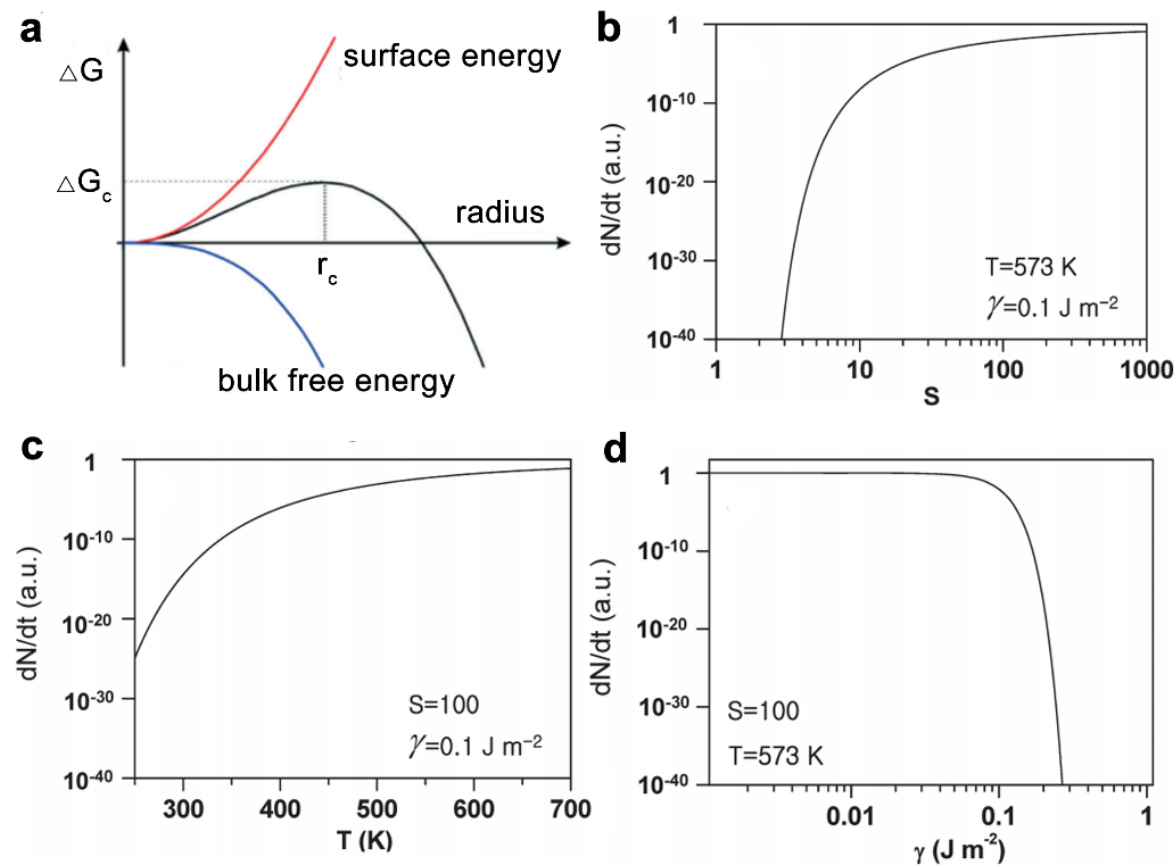

Figure 2. (a) Free energy diagram of nucleus for describing the nucleation process. $\Delta \mathrm{G}$, Gibbs free energy of a nucleus; $\Delta \mathrm{G}_{\mathrm{c}}$, energy barrier; $\mathrm{r}_{\mathrm{c}}$, critical radius of the nucleus with maximum free energy. (b) Nucleation rate as functions of supersaturation. (c) Nucleation rate as function of the temperature. (d) Nucleation rate as function of surface free energy calculated using Eq. (4), which $\mathrm{v}$ is set as $3.29 \times 10^{-5} \mathrm{~m}^{3} \mathrm{~mol}^{-1}$, the value for CdSe NPs. The nucleation rate is normalized with the pre-exponential factor A. Figures are reproduced with permissions from: (a) ref. ${ }^{[19]}$, Copyright 2015, Royal Society of Chemistry; and (b-d) ref. ${ }^{[18]}$, Copyright 2011, Wiley-VCH.

\subsection{Methodology for manipulating the NPs production using microfluidics}

\subsubsection{Alert the flow pattern within the microfluidics chip}

Under a fixed formula for producing specific NPs, the parameters for the synthesized NPs (e.g., size and polydispersity index, PDI) are highly implicated with the mixing efficiency of a microfluidics chip, ${ }^{[30]}$ and the diffusion and convection are the two regimes commonly used in 


\section{WILEY-VCH}

microfluidics that contribute to the mixing efficiency. ${ }^{[31]}$ Diffusion refers to the random motion of solutes driven by a gradient in chemical potential and convection refers to the mixing caused by the convective motion of fluids. ${ }^{[32]}$ In engineering, the behavior of liquids is often described in terms of dimensionless numbers which compare the importance of different physical properties. The Peclect number $(\mathrm{Pe})$ is a commonly applied dimensionless number for mass transfer processes, which relates the rate of advection of a flow to its rate of thermal diffusion. Higher Pe values indicate a more important role of convective bulk flow within the mixing. ${ }^{[33]}$ This can be expressed as Eq. (5): ${ }^{[34]}$

$$
P e=V D / d
$$

where, $\mathrm{V}$ is the total flow speed within the microfluidics channel, $\mathrm{D}$ is the hydraulic crosssectional diameter of the channel and $\mathrm{d}$ is the mass diffusion coefficient of the solute. For a typical microfluidic channel with the a diameter less than $10^{-3} \mathrm{~m}$, a typical Pe number ranging from $10^{2}$ to $10^{4}$ can be observed from two commonly used miscible fluids systems such as ethanol and water, ${ }^{[35]}$ suggesting that convection dominates the mixing procedure. To better understand and explain convection within the flow, considering that the extent of convection is mainly dominated by the fluidic pattern in the microfluidics channel, one considers an additional dimensionless parameter, the Reynolds number $(\mathrm{Re})$, which is defined in Eq. (6): ${ }^{[36]}$

$$
\operatorname{Re}=\rho Q / \eta D=Q / v D
$$

where, $\rho$ is the density of the fluid, $Q$ is the total flow rate of the fluid, $\eta$ is the viscosity of the fluid, $v$ is the kinematic viscosity of the fluid and D is the hydraulic diameter of the channel. Re compares inertial and viscous forces, and a decreased Re always follows that inertia generally becomes unimportant. ${ }^{[32]}$ Considering viscosity produces a resistance to shear and the fluids have a tendency to move in parallel layers, an increase in Re suggests a more chaotic and 


\section{WILEY-VCH}

stochastic fluidic pattern, ultimately resulting in a shift from laminar-to-turbulent flow. As laminar flow only yields fluidic moves in smooth layers, whereas turbulent flows are characterized by chaotic motion of fluid elements and seemingly random fluctuations in instantaneous velocities. Despite the difficulty in predicting and modeling fluid mechanics in turbulent flow, the mixing efficiency within the turbulent regime is vastly enhanced, and is therefore preferred for NPs synthesis.

One method to increase the mixing efficiency is by enhancing the Re within the mixing channel. Kim et al. have long confirmed that increasing values of the Re alter the vortex pattern within the microfluidics channel (Figure 3a) and this further affects the size of the NPs produced (Figure 3b), regardless relatively low maximum values of the $\operatorname{Re}(\operatorname{Re}=150) \cdot{ }^{[37]}$ Farokhzad et al., on the other hand, pushed the Re within the microfluidics channel up to 1311. Different types of NPs, including polymeric NPs (polylactic-co-glycolic acid-polyethylene glycol (PLGA-PEG) and polystyrene (PS), liposomes to metal nanoclusters (iron oxides) were synthesized in a polycarbonate based micro-jetting device. ${ }^{[28]}$ Under a fixed flow rate between inner and outer fluids $\left(\mathrm{R}_{\text {in/out }}\right)$, with the increase of $\mathrm{Re}$, a departure from the laminar flow velocity profile, and the emerging presence of micro-vortex, and ultimately to turbulent jetting, was observed (Figure 3c-e). It should be noted that the conventional transitional $\operatorname{Re}\left(\operatorname{Re}_{t}\right)$, which refers to laminar flow in a circular pipe becomes naturally turbulent at a critical Reynolds number, is usually observed at around 2000 to $2300 .{ }^{[38]}$ However, in microfluidics, a clear turbulent flow can be achieved with a Re lower than $500 .{ }^{[39]}$ Peng et al. first reported that the transition to turbulence occurred at Re as low as $200-700$ in the microchannels with hydraulic diameters of $133-367 \mu \mathrm{m},{ }^{[40]}$ which were similarly observed by other groups. ${ }^{[41]}$ They attributed this phenomenon to the decreased diameter and increased surface roughness of the channel. Readers may refer to an extensive review provided by Gravesen et al. who discussed the Re 


\section{WILEY-VCH}

calculation within microfluidic channels in further detail. ${ }^{[39]}$ Despite the controversial conclusion about the $\mathrm{Re}_{\mathrm{t}}$ within microfluidics channel and corresponding flow pattern, ${ }^{[42]}$ simply by manipulating the flow rate/ratio to alter the Re, one can fabricate NPs with significantly enhanced homogeneity comparing to the bulk method. Previous results suggested that at a total flow rate of $8.3 \mathrm{~mL} \mathrm{~h}^{-1}$, the total mixing of two phases will take place in dozens of seconds. ${ }^{[43]}$ However, the turbulent flow regime, mixing time $\left(t_{\text {mix }}\right)$ is tunable in the range of $7-53$ ms by changing the Re, which is considerably lower than the typical nucleation time ( $\left.\mathrm{t}_{\mathrm{nul}}\right)$ of polymer $\left(\sim 1-100 \mathrm{~ms}\right.$, depending on the molecular weight of the chain) ${ }^{[12,20]}$

Liu et al. further applied a computational fluidic dynamics (CFD) method to illustrate the microfluidics mixing at different flow regime. ${ }^{[26,44]}$ At the flow regime of laminar flow or microvortex flow (Re up to 200), an evaluated mixing efficiency and NPs homogeneity can also be achieved along with the enhanced Re and it is mainly due to an amplified microvortices in the microfluidics channel along with the increase of Re (Figure 3f) ${ }^{[26]}$ With the further increase of $\operatorname{Re}(\operatorname{Re}>500)$, the flow regime will transit to turbulent jet, and due to the ultra-fast flow rate, microscopic observation may fail to detect the violent flow domain changes, ${ }^{[45]}$ and a computational turbulent flow model can be applied to overcome the limitations. ${ }^{[44]}$ As can be seen in Figure 3g, at the $\operatorname{Re}=500$ and 1300, a turbulent flow was simulated, which is indicated by the disappearance of a coaxial jet and complete fluids mixing less than $1 \mathrm{~ms}$, confirming the flow behavior can be feasibly tailored by manipulating the flow rate/ratio. ${ }^{[4]}$

The main advantages for manipulating the Re to control the NPs size/morphology includes, for example, simple fabrication of the chip, easy prediction, and fast operation. Moreover, in the perspective of biomedical applications, the sharply increased flow rate will simultaneously achieve a high throughput NPs production. At the $\mathrm{Re}=200$, a typical glass capillary chip can 


\section{WILEY-VCH}

reach a NPs production rate above $242.8 \mathrm{~g}$ /day, which meets the requirements for industrialscale NPs production, ${ }^{[26,28]}$ hence facilitating industrialization and clinical translation.

However, the accompanied drawbacks mainly come from the ultra-high flow rate induced by this method. In a typical microfluidic channel with the inner diameter of $1 \mathrm{~mm}$, a total flow rate of $5256 \mathrm{~mL} \mathrm{~h}^{-1}$ should be obtained to achieve the Re of 1300 . And the first constraint therefore may come from the maximum force of the fluidic pump, and the corresponding microfluidic device should show suitable pressure resistance. And at this high flow rate, block and stagnation of the channel may cause serious sequential issues. Meanwhile, due to the ultrahigh flow rate, the parameters (such as flow rate, concentration of the starting materials and surfactant etc.) optimization process usually need excessive materials consumption, and therefore may not be suitable for fabricating nanosystems that contain expensive compounds such as proteins, synthetic lipids and/or RNA/DNA. 


\section{WILEY-VCH}

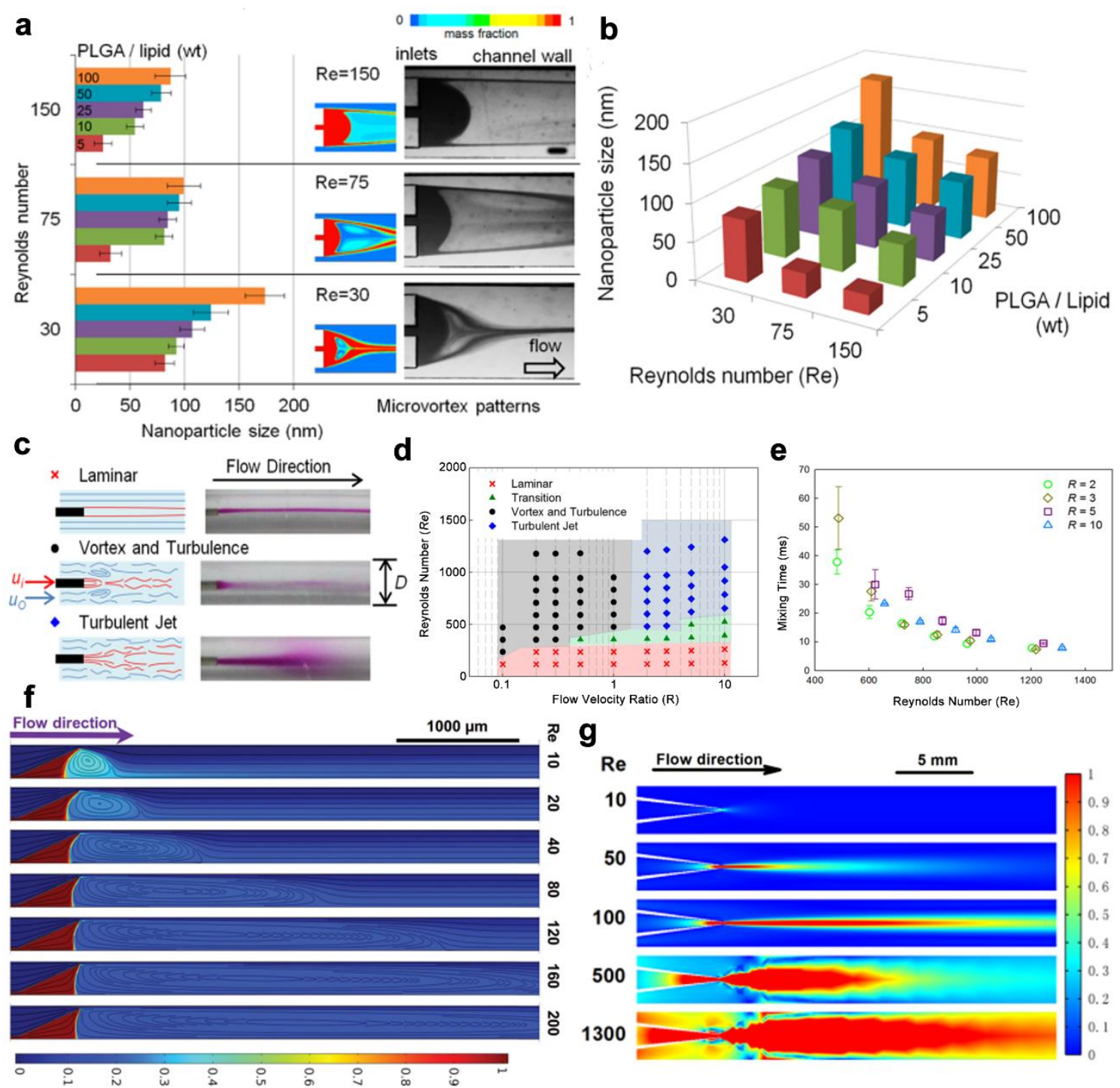

Figure 3. (a) The microvortex within the microchannel can be manipulated by adjusting the parameter values to obtain Re values suitable for NPs production, thus size-controllable NPs syntheses can be achieved through variation of the Re. Microvortex patterns are both predicted by the CFD simulations and visualized by microscopic images. (b) Size map of the produced NPs by varying the Re with given PLGA-to-lipid weight ratios. (c) Macroscopic images of flow pattern alteration obtained by increasing the total flow rate. (d) Phase diagram of the flow regime in terms of flow rate ratio $(\mathrm{R})$ and Re. (e) Quantitative measurement of the reduced mixing time along with the increasing of Re. (f) CFD simulation suggests that within a fixed 


\section{WILEY-VCH}

flow regime (laminar flow), with the enhanced Re, a larger microvortex can be generated in the microchannel, thus inducing a better mixing efficiency. (g) CFD simulation confirmed the ultrahigh mixing efficiency with the flow regime transiting to turbulent flow $(\operatorname{Re}=500,1300)$. Figures are reproduced with permissions from: (a-b) ref. ${ }^{[37]}$, Copyright 2012, American

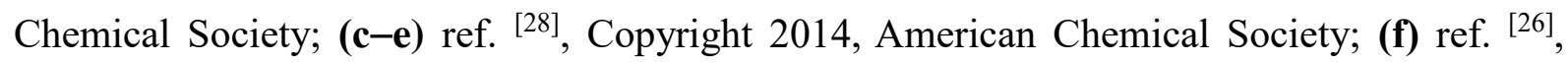
Copyright 2015, Wiley-VCH; (g) ref. ${ }^{[44]}$, Copyright 2017, American Chemical Society.

\subsubsection{Alter the geometry of the mixing channel}

The other commonly used method is typically based upon smart design of the channel geometries. Various types of microfluidic chips with complex geometry, such as Tesla-shape, ${ }^{[46]}$ herringbone-shaped ${ }^{[47,48]}$ serpentine-shaped, ${ }^{[49]}$, planar asymmetric split, ${ }^{[50]}$ spiral/semispiral, ${ }^{[51,52]}$ zigzag-shaped channel etc., ${ }^{[53]}$ were designed and applied for preparing NPs (Figure 4a-e). For example, herringbone-shaped microchannel containing patterned microgrooves of varying shapes and angles can induce chaotic stirring at a low $\operatorname{Re}\left(\operatorname{Re}=10^{-2}\right.$ $\left.10^{2}\right)$. At relatively low total flow rate $\left(600 \mu \mathrm{L} \mathrm{min}^{-1}\right)$, the efficient mixing time remains on the order of milliseconds ( $\sim 8 \mathrm{~ms})$ by applying this apparatus. ${ }^{[48]}$ Complex geometry can decrease the mixing length at relatively low Re, thus achieved a thorough mixing at low flow rate, therefore more suitable for precise production of costly NPs. For example, Tokeshi et al. recently applied a multi-baffles integrated microfluidics chip to produce siRNA-loaded liposomes. The newly designed chip showed a non-inferior mixing efficiency compared to herringbone-shaped chip (fully mixing at the flow rate of $500 \mu \mathrm{L} \mathrm{min}^{-1}$ ). More importantly, for the first time it was reported liposomes size tuning at $10 \mathrm{~nm}$ intervals in the size range from 20 to $100 \mathrm{~nm}$ (Figure $4 \mathbf{f}) \cdot{ }^{[54]}$ Not only by designing the geometry of the mixing chamber, Bokare et al. reported that even by simply change the geometry pattern of the inlet channel for the 


\section{WILEY-VCH}

fluids, the mixing efficiency can also be affected, as by integrating the herringbone-pattern with the inlet channel of a micro-cortex mixer (Figure 4g), the size of produced PLGA/lipid nanohybrids reduced from $199 \mathrm{~nm}$ to $74.5 \mathrm{~nm} .^{[55]}$

Besides of influencing the size and morphology of NPs, as the altered geometry of microfluidic chips may impact on the mixing efficiency and shear force of the fluids, ${ }^{[56]}$ thus the geometry of microfluidic chips may also dictate the internal crystallinities of the NPs. Moffitt et al. investigated the size, morphologies and crystallinities of polycaprolactone-block-poly(ethylene oxide) (PCL- $b$-PEO) NPs produced by single-phase staggered herringbone (SHB) mixer (featured with high mixing rate, but low shear force) or two-phase gas-liquid segmented mixer (featured with low mixing rate, but high shear force). ${ }^{[57]}$ The results suggested that the morphologies, dimensions, and crystallinities of NPs produced in the single-phase SHB and two-phase mixer was similar in low flow rate regime $\left(20 \mu \mathrm{L} \mathrm{min} \operatorname{mit}^{-1}\right)$, but showed a different feature at high flow rate regime $\left(60\right.$ and $\left.100 \mu \mathrm{L} \mathrm{min}{ }^{-1}\right)$, while the crystallinities of the NPs produced in the bulk method (featured with low mixing rate and low shear force) are similar with NPs produced in SHB (Figure 4h). These finding suggested that the mixing rate strongly affects the NPs morphologies and dimensions, and the mixing rate has a much weaker effect on the core crystallinities, which are mainly influenced by shear effects.

One main disadvantage of the complex chip geometry design is that the chip usually involves subtle and precise channels. Therefore, the channels may easily get clogged during the NPs production, which leads to stagnation of the sample flow. ${ }^{[54]}$ In addition, the accurate chip design usually lacks flexibility and robustness, especially regarding the microchannel aspect ratio when fabricating three-dimensional mixer structures. ${ }^{[54]}$ Furthermore, to fabricate a complex geometry, the most commonly used material for preparing the microfluidic chips is 


\section{WILEY-VCH}

polydimethylsiloxane (PDMS). PDMS is not resistant to many organic solvents, which may constrain its potential application in the producing of NPs ${ }^{[58]}$ For glass or silicon chips, despite their chemical inertness and optically transparent feature, standard photolithography to produce such devices is usually involved in dangerous chemicals (e.g., hydrofluoric acid). Also harsh conditions, including high temperature, high pressure and super clean environment, are commonly required for the bonding process. ${ }^{[59]}$ As such, posing limits to their broad applications.

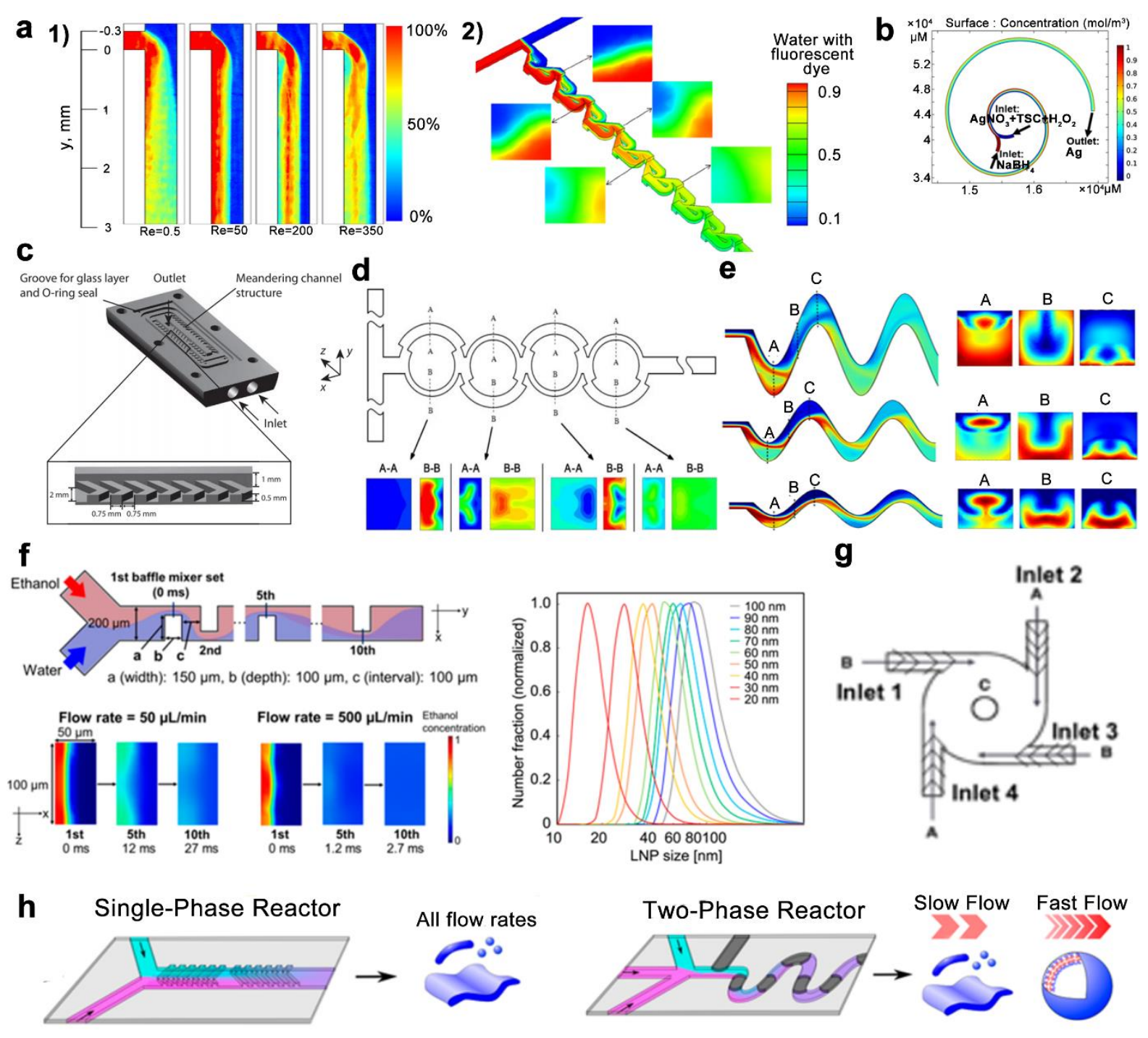

Figure 4. (a-e) Representative microfluidic chips with complex geometry used to achieve a thorough mixing at low flow rate. (a1) Conventional T-shape microfluidic chip showed a 


\section{WILEY-VCH}

limited mixing efficiency at various flow rate, and (a2) Tesla-shaped microfluidic chip can enhance the mixing performance for Re ranging from 0.1 to $100\left(0.015-15 \mu \mathrm{L} \mathrm{s}^{-1}\right)$. (b) Typical serpentine structured microfluidic chips for producing silver nanoparticles. (c) A typical microfluidic chip with patterned staggered herringbone geometry. (d) Mass-fraction distributions at $\mathrm{Re}=80$ by applying planar asymmetric split and recombine microfluidic chip. (e) The mixing efficiency of zigzag-shape microfluidics chip can be altered by changing the amplitude ratio-to-wave length at a fixed inlet Re of 50. (f) Schematic illustration of the multibaffles integrated microfluidics chip and CFD simulation results for the flow rates of 50 and $500 \mu \mathrm{L} \mathrm{min}{ }^{-1}$. The application of the current chip can precisely control the produced liposomes at $10 \mathrm{~nm}$ intervals in the size range from 20 to $100 \mathrm{~nm}$ (right panel). (g) Geometry pattern of the inlet channel can also be modified to achieve better mixing efficiency. (h) The geometry pattern of the microfluidic can also affect the crystalline and morphology of the produced nanomicelle. Figures are reproduced with permissions from: (a1) ref. ${ }^{[60]}$, Copyright 2012, Elsevier;

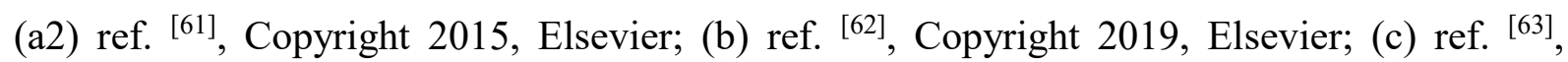
Copyright 2018, Elsevier; (d) ref. ${ }^{[50]}$, Copyright 2013, Wiley-VCH; (e) ref. ${ }^{[64]}$, Copyright 2014, Elsevier; (f) ref. ${ }^{[54]}$, Copyright 2018, American Chemical Society; (g) ref. ${ }^{[55]}$, Copyright 2019, American Chemical Society; and (h) ref. ${ }^{[57]}$, Copyright 2016, American Chemical Society.

\subsubsection{External energy integrated microfluidic chips}

So far, efforts have been made to enhance the mixing in microfluidics by integrating outer sources of energy. The general idea is to introduce an external energy to agitate the fluid, as such enhancing the mixing efficiency.

Ultrasonic/acoustic vibrations are commonly applied energy sources to generate turbulence in 


\section{WILEY-VCH}

the fluid. Microfluidics chips can be integrated with piezoelectric ceramics, which operate at a high frequency in the $\mathrm{kHz}$ region, to locally generate pressure fluctuations in liquids and disturb the laminar flow pattern. ${ }^{[65]} \mathrm{A}$ better mixing efficiency can be achieved with a higher frequency or voltage excitation, however, it may induce unintended heating of the fluid. Yet it takes a few seconds to tens of seconds to achieve homogenous mixing. ${ }^{[66]}$ Further methods applied sidewall trapped microbubbles as a piezo transducer to form microstreaming, and successfully reduced the mixing time to less than $120 \mathrm{~ms}$ at a driving frequency of $81.4 \mathrm{kHz}$ with the flow rate of $3 \mu \mathrm{L} \mathrm{min}^{-1}{ }^{167]}$ Surface acoustic wave-powered microfluidic chips offer an alternative acoustic streaming source, which can further reduce the mixing time to $11 \mathrm{~ms}^{[68]}$

Other methods, such as applying pressure perturbation, dielectrophoresis induced dipole moment, pulsed/periodic electrodynamics actuation, and optics can extensively enhance the mixing efficiency, and thus, have been widely applied in biological samples' analysis and biosynthesis, and readers may refer to an extensive review by Lee et al. for further information. ${ }^{[29]}$ However, the fabrication of the corresponding chips is relatively complicated and expensive, and in addition the successful mixing usually needs the fluids to obtain special electrical properties, hence have been relatively less applied in the NPs fabrication.

\subsection{From simple to core/shell fabrication}

\subsubsection{Synthesis and application of microfluidic-produced core/shell NPs}

There is a rich literature that provides reviews that focus on several aspects of the applications of microfluidics produced NPs for biomedical usages. For example, Luo et al. and Swider et al. reviewed the specific NPs productions within microfluidics such as inorganic NPs and PLGA NPs ${ }^{[69]}$ Ahn et al., Colombo et al. reviewed the role of microfluidics in promoting the clinical 


\section{WILEY-VCH}

translation of nanomedicine. ${ }^{[70]}$ Sanjay et al. and Liu et al. summarized the application of microfluidics in synthesizing advanced drug release systems. ${ }^{[71]}$ For the sake of brevity, the current review will mainly focusing on the NPs with identical core/shell structures and their corresponding applications.

After the initial research work that mainly focused on using microfluidics to produce basic NPs for multiple biomedical applications, recent efforts have been made to fabricate nanohybrids that incorporate two or more nanomaterials. ${ }^{[72]}$ Comparing to the conventional nanosystems, nanohybrids exhibit enhanced biocompatibility, stability, catalytic properties and hierarchical control. ${ }^{[73]}$ Among which, nanohybrids with identical core/shell structures, such as organic/inorganic nanohybrids, ${ }^{[74]}$ inorganic/inorganic nanohybrids ${ }^{[75]}$ and lipid/polymer nanohybrids ${ }^{[52]}$ have been widely investigated. The previous bulk methods used to produce core/shell nanosystems usually contains two independent steps to separately prepare the core structure and the sequential shell coating, and thus often exhibit poor control over the encapsulation efficiency and reproducibility. With the development of microfluidics, a continuous method combining the simultaneous and/or sequential nanoprecipitation of core NPs and shell coating showed considerable advantages by reducing intermediate disturbances and batch-to-batch variation. ${ }^{[76]}$ Tables $\mathbf{1}$ and $\mathbf{2}$ briefly list the examples of the microfluidics used to produce core/shell structures over the past 5 years. In the following text we will highlight some of the cases.

Table 1: Examples of single-step precipitation methods for producing core/shell NPs investigated from 2014-2019.

\begin{tabular}{cccccc}
$\begin{array}{c}\text { Core } \\
\text { Materials }\end{array}$ & Shell Materials & $\begin{array}{c}\text { Inlet Fluid 1 } \\
(\text { F1 })\end{array}$ & $\begin{array}{c}\text { Inlet Fluid 2 } \\
(\text { F2 })\end{array}$ & $\begin{array}{c}\text { Flow Rate Ratio } \\
(\text { F1 :F2) }\end{array}$ & References \\
\cline { 5 - 6 } & & &
\end{tabular}


WILEY-VCH

\begin{tabular}{|c|c|c|c|c|c|}
\hline $\mathrm{Fe}_{3} \mathrm{O}_{4} \mathrm{NPs}$ & $\begin{array}{c}\text { Purified red } \\
\text { blood cells } \\
\text { (RBC) vehicles }\end{array}$ & $\begin{array}{l}0.4 \mathrm{mg} \mathrm{mL}^{-1} \\
\mathrm{Fe}_{3} \mathrm{O}_{4} \mathrm{NPs} \text { in } \\
\mathrm{PBS} \text { buffer }\end{array}$ & $\begin{array}{l}2.5 \mathrm{~mL} \text { PBS } \\
\text { containing } \\
\text { RBC-vehicle } \\
\text { from } 0.2 \mathrm{~mL} \\
\text { of mouse } \\
\text { blood }\end{array}$ & $20 \mu \mathrm{L} \min _{\min ^{-1}}^{-1}: 20 \mu \mathrm{L}$ & [77] \\
\hline $\mathrm{Fe}_{3} \mathrm{O}_{4} \mathrm{NPs}$ & Au NPs & $\begin{array}{c}21-126 \mu \mathrm{g} \\
\mathrm{mL}^{-1} \text { as- } \\
\text { prepared } \\
\mathrm{Fe}_{3} \mathrm{O}_{4} \mathrm{NPs} \text { in } \\
\mathrm{H}_{2} \mathrm{O}\end{array}$ & $\begin{array}{l}\text { As prepared } \\
\text { Au NPs } \\
\text { (containing } \\
1.5 \mathrm{mM} \text { ) in } \\
\mathrm{H}_{2} \mathrm{O}\end{array}$ & $100 \mu \mathrm{L} \min _{\min ^{-1}}: 100 \mu \mathrm{L}$ & [78] \\
\hline $\mathrm{Fe}_{2} \mathrm{O}_{3} \mathrm{NPs}$ & PLGA & $\begin{array}{c}\text { PLGA (5 mg } \\
\left.\mathrm{mL}^{-1}\right) \text { and } \\
\mathrm{Fe}_{2} \mathrm{O}_{3} \mathrm{NPs} \text { in } \\
\text { tetrahydrofura } \\
\mathrm{n}(\mathrm{THF})\end{array}$ & $\mathrm{H}_{2} \mathrm{O}$ & n.s. & [79] \\
\hline $\begin{array}{l}\text { Spermine } \\
\text { acetalated } \\
\text { dextran } \\
\text { (AcDEX) } \\
\text { modified } \\
\text { porous silicon } \\
\text { (PSi) NPs, } \\
\text { spermine } \\
\text { AcDEX } \\
\text { modified Au } \\
\text { NPs }\end{array}$ & AcDEX & $\begin{array}{c}\text { PSi }(0.5 \mathrm{mg} \\
\mathrm{mL}^{-1)}, \mathrm{Au} \mathrm{NPs}^{-1} \\
\left(2.5 \mathrm{mg} \mathrm{mL}^{-1}\right) \\
\text { were dispersed } \\
\text { into the } \\
\text { AcDEX }(10 \\
\left.\mathrm{mg} \mathrm{mL}^{-1}\right) \\
\text { ethanol/aceton } \\
\text { itrile (90:10, } \\
\text { v/v, with } 0.2 \% \\
\text { TEA) }\end{array}$ & $\begin{array}{c}1 \% \\
\text { Poloxamer- } \\
188(\mathrm{P}-188) \\
\mathrm{H}_{2} \mathrm{O} \text { solution }\end{array}$ & $1 \mathrm{~mL} \mathrm{~h}^{-1}: 20 \mathrm{~mL} \mathrm{~h}^{-1}$ & [80] \\
\hline PSi NPs & $\begin{array}{c}\text { 4- } \\
\text { (hydroxymethyl } \\
\text { )-phenylboronic } \\
\text { acid pinacol } \\
\text { ester conjugated } \\
\text { with oxidized } \\
\text { dextran (POD) }\end{array}$ & $\begin{array}{l}\mathrm{PSi}\left(1 \mathrm{mg} \mathrm{mL}^{-}\right. \\
1) \text { dispersed } \\
\text { into POD }(15 \\
\left.\mathrm{mg} \mathrm{mL}^{-1}\right) \text { in } \\
\text { ethanol/ } \mathrm{H}_{2} \mathrm{O} \\
(80 / 20, \mathrm{v} / \mathrm{v})\end{array}$ & $\mathrm{H}_{2} \mathrm{O}$ & $1 \mathrm{~mL} \mathrm{~h}^{-1}: 10 \mathrm{~mL} \mathrm{~h}^{-1}$ & [81] \\
\hline $\begin{array}{l}\text { Sorafenib/It } \\
\text { raconazole } \\
\text { Nanocrystal } \\
\text { s }\end{array}$ & AcDEX & $\begin{array}{l}\text { As-prepared } \\
\text { drug } \\
\text { nanocrystals } \\
\text { in saturated } \\
\text { ethanol } \\
\text { solution } \\
\text { containing } 5 \\
\text { mg mL } \\
\text { acetalated } \\
\text { dextran }\end{array}$ & $\mathrm{H}_{2} \mathrm{O}(\mathrm{pH}=8)$ & n.s. & [75] \\
\hline PSi NPs & AcDEX & $\begin{array}{c}0.1-2 \mathrm{mg} \mathrm{mL}^{-} \\
1 \text { of PSi NPs } \\
\text { dispersed in } \\
1-10 \mathrm{mg} \mathrm{mL}^{-1} \\
\text { AcDEX } \\
\text { ethanol } \\
\text { solution }\end{array}$ & $\begin{array}{l}\text { PVAV(MW } \\
31-50 \mathrm{kDa}, 1 \\
\left.\mathrm{mg} \mathrm{mL}^{-1}\right) \\
\text { aqueous } \\
\text { solution }\end{array}$ & $3 \mathrm{~mL} \mathrm{~h}^{-1}: 100 \mathrm{~mL} \mathrm{~h}^{-1}$ & [82] \\
\hline $\begin{array}{l}\text { Prickly Zn- } \\
\text { CuO NPs }\end{array}$ & $\begin{array}{l}\text { Spermine } \\
\text { AcDEX }\end{array}$ & $\begin{array}{l}0.5 \mathrm{mg} \mathrm{mL}^{-1} \text { of } \\
\text { prickly Zn- } \\
\text { CuO NPs } \\
\text { mixed with } \\
2.5 \mathrm{mg} \mathrm{mL}^{-1} \\
\text { of Spermine } \\
\text { AcDEX in } \\
\text { ethanol }\end{array}$ & $\begin{array}{l}1 \% \text { PVA ( } \mathrm{pH} \\
\text { 8) aqueous } \\
\text { solution }\end{array}$ & $2 \mathrm{~mL} \mathrm{~h}^{-1}: 40 \mathrm{~mL} \mathrm{~h}^{-1}$ & [83] \\
\hline
\end{tabular}


WILEY-VCH

\begin{tabular}{|c|c|c|c|c|c|}
\hline $\begin{array}{l}\text { Poly-o- } \\
\text { phenylenedia } \\
\text { mine (P- } \\
\text { OPD) NPs }\end{array}$ & $\mathrm{Ag}$ & $\begin{array}{l}0.02 \mathrm{M} \text { POPD } \\
\text { aqueous } \\
\text { solution }\end{array}$ & $\begin{array}{l}0.01 \mathrm{M} \\
\mathrm{AgNO}_{2} \\
\text { aqueous } \\
\text { solution }\end{array}$ & $\begin{array}{c}15-60 \mu \mathrm{L} \min ^{-} \\
1: 30-60 \mu \mathrm{L} \mathrm{min}{ }^{-1}\end{array}$ & [84] \\
\hline $\begin{array}{l}\text { Eutectic } \\
\text { gallium } \\
\text { indium } \\
\text { (EGaIn) NPs }\end{array}$ & $\begin{array}{c}\text { Trithiocarbonate } \\
\text {-functionalized } \\
\text { Brushed } \\
\text { polyethylene } \\
\text { glycol (bPEG, } \\
\text { MW 5-20 kDa) }\end{array}$ & $\begin{array}{l}\text { EGaln } \\
\text { solution }\end{array}$ & $\begin{array}{l}0.5 \mathrm{M} \mathrm{bPEG} \\
\text { aqueous } \\
\text { solution }\end{array}$ & $1 \mu \mathrm{L} \min _{\min ^{-1}}: 50 \mu \mathrm{L}$ & [85] \\
\hline $\begin{array}{l}\text { Poly-methyl } \\
\text { methacrylate } \\
\text { (PMMA) NPs }\end{array}$ & $\begin{array}{c}\text { Superparamagn } \\
\text { etic iron oxide } \\
\text { nanoparticles } \\
\text { (SPIONs, } \sim 6 \\
\text { nm) }\end{array}$ & $\begin{array}{c}\text { PMMA (50 } \\
\text { mg, } \\
\text { Cremophor } \\
\text { ELP (25 mg) } \\
\text { mixed with } 5 \\
\text { mL of the } \\
\text { dispersion of } \\
\text { SPIONs in } \\
\text { tetrahydrofura } \\
\text { n (THF) to } \\
\text { adjusted to } 15 \\
\text { mg SPIONs } \\
\text { per mL }\end{array}$ & $\mathrm{H}_{2} \mathrm{O}$ & $0.2 \mathrm{~mL} \min _{\min ^{-1}}^{-1}: 2-4 \mathrm{~mL}$ & [74] \\
\hline $\begin{array}{l}\text { Chitosan- } \\
\text { modified PSi }\end{array}$ & $\begin{array}{l}\text { Hypromellose } \\
\text { acetate } \\
\text { succinate }(\mathrm{HF})\end{array}$ & $\begin{array}{l}\text { Chitosan- } \\
\text { modified PSi } \\
\text { dispersed in } \\
10 \mathrm{mg} \mathrm{mL}^{-1} \\
\text { HF solution in } \\
\text { acetone }\end{array}$ & $\begin{array}{c}1 \% \text { PVA } \\
\text { aqueous } \\
\text { solution, } \mathrm{pH} \\
3.7 .\end{array}$ & $2 \mathrm{~mL} \mathrm{~h}^{-1}: 40 \mathrm{~mL} \mathrm{~h}^{-1}$ & [86] \\
\hline $\begin{array}{c}\text { Oleic acid } \\
\text { capped } \mathrm{Fe}_{2} \mathrm{O}_{3} \\
\mathrm{NPs}\end{array}$ & Lipid & $\begin{array}{c}\mathrm{Fe}_{2} \mathrm{O}_{3} \mathrm{NPs} \text {, } \\
\text { triolein, 1- } \\
\text { palmitoyl-2- } \\
\text { oleoyl-sn- } \\
\text { glycero-3- } \\
\text { phosphocholin } \\
\text { e (POPC), and } \\
\text { 1,2-distearoyl- } \\
\text { sn-glycero-3- } \\
\text { phosphoethan } \\
\text { olamine-N- } \\
\text { [methoxy- } \\
\text { (polyethylene } \\
\text { glycol)-2000] } \\
\text { (DSPE-PEG) } \\
\text { mixed at a } \\
\text { molar ratio of } \\
72: 25: 3 \\
\text { (respectively) } \\
\text { to a final lipid } \\
\text { concentration } \\
\text { of } 13.6 \text { mM in } \\
\text { a solvent } \\
\text { mixture (10.16 } \\
\mu \text { mol lipid per } \\
\text { mg Fe } 2 \mathrm{O}_{3} \text { ) } \\
\text { containing at } \\
\text { least } 40 \% \\
\text { THF in } \\
\text { ethanol. }\end{array}$ & $\mathrm{H}_{2} \mathrm{O}$ & $5 \mathrm{~mL} \min _{\min ^{-1}}^{-1}: 15 \mathrm{~mL}$ & [87] \\
\hline $\begin{array}{c}\text { Graphene } \\
\text { oxide (GO) } \\
\text { NPs }\end{array}$ & $\begin{array}{l}\text { 1,2-dioleoyl-3- } \\
\text { trimethylammon } \\
\text { ium-propane } \\
\text { (DOTAP) }\end{array}$ & $\begin{array}{l}30 \mu \mathrm{g} \mathrm{mL}^{-1} \\
\mathrm{GO} \mathrm{NPs}^{2}\end{array}$ & $\begin{array}{l}\text { DOTAP } \\
\text { cationic } \\
\text { liposomes } \\
\text { with lipid }\end{array}$ & $12 \mathrm{~mL} \min _{\min ^{-1}}^{-1}: 12 \mathrm{~mL}$ & [88] \\
\hline
\end{tabular}


n.a.: not applicable; n.s.: not specified.

Table 2: Examples of sequential precipitation methods for producing core/shell NPs investigated from year 2014-2019.

\begin{tabular}{|c|c|c|c|c|c|c|c|}
\hline \multirow{2}{*}{$\begin{array}{c}\text { Core } \\
\text { Materia } \\
\quad \text { ls }\end{array}$} & \multicolumn{2}{|c|}{ First Mixing } & \multirow{2}{*}{$\begin{array}{c}\text { Shell } \\
\text { Materia } \\
\text { ls }\end{array}$} & \multicolumn{2}{|c|}{ Second Mixing } & \multirow{2}{*}{$\begin{array}{c}\text { Flow Rate } \\
\text { (F1:F2:F3 } \\
\text { :F4) }\end{array}$} & \multirow{2}{*}{$\begin{array}{c}\text { Refere } \\
\text { nces }\end{array}$} \\
\hline & Fluid 1 (F1) & Fluid 2 (F2) & & $\begin{array}{c}\text { Fluid } 3 \\
\text { (F3) }\end{array}$ & $\underset{\text { (F4) }}{\text { Fluid } 4}$ & & \\
\hline $\begin{array}{l}\text { Cobalt } \\
\text { (Co) } \\
\text { NPs }\end{array}$ & $\begin{array}{c}8 \mathrm{mM} \mathrm{CoCl}_{2} \\
\text { in } 8 \mathrm{mM} \text { citric } \\
\text { buffer }\end{array}$ & $\begin{array}{c}0.1 \mathrm{wt}-\% \text { PVP } \\
(55 \mathrm{kDa})+ \\
19.2 \mathrm{mM} \\
\mathrm{NaBH}_{4} \text { in } \mathrm{H}_{2} \mathrm{O}\end{array}$ & Gold & $\begin{array}{c}0.5 \mathrm{mM} \\
\mathrm{HAuCl}_{4} \\
\text { water } \\
\text { solution }\end{array}$ & n.a. & $\begin{array}{c}15 \mathrm{~mL} \mathrm{~h}^{-} \\
1: 15 \mathrm{~mL} \mathrm{~h}^{-} \\
1: 25 \mathrm{~mL} \mathrm{~h}^{-1}\end{array}$ & [89] \\
\hline $\begin{array}{l}\text { Titaniu } \\
\text { m NPs }\end{array}$ & $\begin{array}{c}0.458 \mathrm{~mL} \\
\text { titanium } \\
\text { tetraisopropox } \\
\text { ide (TTIP) in } \\
9.44 \mathrm{~g} \\
\text { isopropyl } \\
\text { alcohol (IPA) }\end{array}$ & $\begin{array}{c}0.078 \mathrm{~g} \mathrm{H}_{2} \mathrm{O}+ \\
\quad 9.74 \mathrm{~g} \mathrm{IPA}\end{array}$ & $\mathrm{SiO}_{2}$ & $\begin{array}{l}1.148 \mathrm{~g} \text { of } \\
\text { ethylsilicate } \\
\text { (TEOS) in } \\
8.7 \mathrm{~g} \mathrm{IPA}\end{array}$ & $\begin{array}{c}0.758 \mathrm{~g} \\
\text { ammonia in } \\
2.84 \mathrm{~g} \mathrm{H}_{2} \mathrm{O} \\
\text { and } 6.98 \mathrm{~g} \\
\text { IPA }\end{array}$ & n.s. & [90] \\
\hline $\begin{array}{l}\text { PLGA } \\
\text { NPs }\end{array}$ & $\begin{array}{c}4.55 \mathrm{mg} \mathrm{mL}^{-1} \\
1,2- \\
\text { dipalmitoyl- } \\
\text { sn-glycero-3- } \\
\text { phosphocholin } \\
\text { e (DPPC), } \\
0.48 \mathrm{mg} \mathrm{mL}^{-1} \\
\text { cholesterol, } \\
0.48 \mathrm{mg} \mathrm{mL}^{-1} \\
\text { DSPE- } \\
\text { PEG2000 }\end{array}$ & $\mathrm{H}_{2} \mathrm{O}$ & $\begin{array}{l}\text { Liposo } \\
\text { mes }\end{array}$ & $\begin{array}{l}100 \mathrm{mg} \\
\text { PLGA in } \\
6.5 \mathrm{~mL} \\
\text { dimethylfor } \\
\text { mamide } \\
\text { (DMF), and } \\
3.5 \mathrm{~mL} \text { of } \\
\text { trifluoroeth } \\
\text { anol (TFE) }\end{array}$ & n.a. & $\begin{array}{c}0.5 \mathrm{~mL} \mathrm{~h}^{-} \\
1: 40 \mathrm{~mL} \mathrm{~h}^{-} \\
{ }^{1}: 0.5 \mathrm{~mL} \mathrm{~h}_{1}^{-}\end{array}$ & [91] \\
\hline $\begin{array}{l}\text { PLGA } \\
\text { NPs }\end{array}$ & $\begin{array}{l}100 \mathrm{mg} \text { PLGA } \\
\text { in } 6.5 \mathrm{~mL} \\
\text { DMF, and } \\
3.5 \mathrm{~mL} \text { of } \\
\text { trifluoroethano } \\
1 \text { (TFE) }\end{array}$ & $\mathrm{H}_{2} \mathrm{O}$ & $\begin{array}{l}\text { Lipid } \\
\text { mono } \\
\text { layer }\end{array}$ & $\begin{array}{c}4.55 \mathrm{mg} \\
\mathrm{mL}^{-1} \mathrm{DPPC} \\
0.48 \mathrm{mg} \\
\mathrm{mL}^{-1} \\
\text { cholesterol, } \\
0.48 \mathrm{mg} \\
\mathrm{mL}^{-1} \\
\text { DSPE- } \\
\text { PEG2000 }\end{array}$ & n.a. & $\begin{array}{c}0.5 \mathrm{~mL} \mathrm{~h}^{-} \\
1: 40 \mathrm{~mL} \mathrm{~h}^{-} \\
1: 0.5 \mathrm{~mL} \mathrm{~h}_{1}^{-}\end{array}$ & [52] \\
\hline $\mathrm{H}_{2} \mathrm{O}$ & $\begin{array}{c}\text { PLGA and } \\
\text { 1,2-dioleoyl- } \\
3- \\
\text { trimethylamm } \\
\text { onium- } \\
\text { propane } \\
\text { (DOTAP) in } \\
\text { DMF and } \\
\text { TFE (65/35, } \\
\text { v/v) at a mass } \\
\text { ratio of } 25: 3\end{array}$ & $\mathrm{H}_{2} \mathrm{O}$ & $\begin{array}{l}\text { PLGA/ } \\
\text { Lipid } \\
\text { hybrids }\end{array}$ & $\begin{array}{c}\text { DPPC, } \\
\text { DSPE-PEG } \\
\text { and } \\
\text { cholesterol } \\
\text { in ethanol } \\
\text { with molar } \\
\text { ratio of } 80: \\
4: 16\end{array}$ & n.a. & $\begin{array}{c}2 \mathrm{~mL} \mathrm{~h}^{-} \\
1: 30 \mathrm{~mL} \mathrm{~h}^{-} \\
1: 0.5 \mathrm{~mL} \mathrm{~h}^{-}\end{array}$ & [92] \\
\hline
\end{tabular}




\section{WILEY-VCH}

\begin{tabular}{|c|c|c|c|c|c|c|c|}
\hline $\begin{array}{l}\text { Silver } \\
\text { NPs }\end{array}$ & $\begin{array}{c}8 \mathrm{mM} \mathrm{CuSO}_{4} \\
\text { in } \mathrm{H}_{2} \mathrm{O}\end{array}$ & $\begin{array}{c}0.6-2 \mathrm{M} \\
\mathrm{NaOH} \text { in } \mathrm{H}_{2} \mathrm{O}\end{array}$ & $\mathrm{CuO}_{2}$ & $\begin{array}{c}\text { As-prepared } \\
\mathrm{Ag} \\
\text { nanoprisms } \\
\text { in } \mathrm{H}_{2} \mathrm{O}\end{array}$ & $\begin{array}{l}1.9-5.7 \mathrm{mM} \\
\text { ascorbic } \\
\text { acid in } \mathrm{H}_{2} \mathrm{O}\end{array}$ & $\begin{array}{c}0.25 \mathrm{~mL} \\
\min ^{-1}: 0.25 \\
\mathrm{~mL} \min ^{-} \\
{ }^{1}: 0.5 \mathrm{~mL} \\
\min ^{-1}: 0.5 \\
\mathrm{~mL} \mathrm{~min}^{-1}\end{array}$ & [93] \\
\hline $\begin{array}{c}\text { Sorafeni } \\
\text { b/ } \\
\text { Paclitax } \\
\text { el } \\
\text { Nanocry } \\
\text { stals }\end{array}$ & $\begin{array}{l}\text { Drug acetone } \\
\text { solution }(10 \\
\left.\mathrm{mg} \mathrm{mL}^{-1}\right) \\
\text { containing HF, } \\
\left(10 \mathrm{mg} \mathrm{mL}^{-1}\right)\end{array}$ & $\begin{array}{c}\text { Basic aqueous } \\
\text { solution (pH } \\
10.4)\end{array}$ & $\mathrm{HF}$ & $\begin{array}{l}\text { Acidic } \\
\text { aqueous } \\
\text { solution } \\
\text { (pH 3) }\end{array}$ & n.a. & $\begin{array}{c}4.05 \times 10^{-8} \\
\mathrm{~m}^{3} \mathrm{~s}^{-} \\
1: 2.02 \times 10^{-7} \\
\mathrm{~m}^{3} \mathrm{~s}^{-} \\
{ }^{-}: 1.21 \times 10^{-6} \\
\mathrm{~m}^{3} \mathrm{~s}^{-1}\end{array}$ & [44] \\
\hline Chitosan & $\begin{array}{l}\text { Aqueous } \\
\text { solution of } \\
\text { chitosan with } \\
\text { the pH of } 5.5\end{array}$ & $\begin{array}{l}\text { Basic aqueous } \\
\quad(\mathrm{pH} \text { of } 9)\end{array}$ & $\underset{\mathrm{t}}{\text { Eudragi }}$ & $\begin{array}{c}\text { Eudragit FS } \\
\text { 30D (0.2 } \\
\text { wt-\%) in } \\
\text { ethanol/ } \\
\text { deionized } \\
\text { water } \\
\text { solution }\end{array}$ & n.a. & $\begin{array}{l}\text { For F1:F2, } \\
\text { volumetric } \\
\text { ratio of } \\
\text { sheath } \\
\text { flow to } \\
\text { main flow } \\
\text { from } 0.03 \\
\text { to } 0.3 \text {. } \\
\text { Flow ratio } \\
\text { of F3 } \\
\text { changed } \\
\text { from } 0.07 \\
\text { to } 0.3 \text {, } \\
\text { while } \\
\text { keeping } \\
\text { the flow } \\
\text { ratio } \\
\text { constant } \\
\text { for F1 and } \\
\text { F2 }(0.03)\end{array}$ & [94] \\
\hline
\end{tabular}

n.a.: not applicable; n.s.: not specified.

Changes targeting the composition of the fluidic channels suffice to achieve multiple hierarchical structures. Jiang et al. applied a two-stage microfluidic platform, which was composed of two consecutive nanoprecipitation devices (Figure 5a-b). ${ }^{[52,91,92]}$ First, a PLGA solution is introduced into the first precipitation stage and lipid solution is injected into the second stage. Secondly, a PLGA/lipid-layer core/shell structure is formed by injecting lipid solution into the first precipitation stage, and the PLGA solution into the second stage in order to form the PLGA/liposome structure. The difference of their structure was confirmed by cryotransmission electron microscope (Cryo-TEM) and fluorescence quenching titration method. Computational molecular dynamics simulation was used to illustrate the encapsulation process, suggesting the composition of shell materials directly dictated the cellular uptake of the NPs, 


\section{WILEY-VCH}

as such highlighting the consequence of changing shell materials in manipulating the physiochemical properties of the NPs.

Based on this idea, core/shell structures were widely applied into encapsulating pre-existed NPs to improve their stability, biocompatibility and targeting ability. For example, Zhang et al. encapsulated prickly zinc-doped copper oxide nanoparticles with a carbonic anhydrase IX targeting ligand modified spermine-acetalated dextran (Spermine-AcDEX). ${ }^{[83]}$ Comparing to the pristine NPs, the newly produced core/shell nanohybrids obtained a better human plasma stability (aggregation time $5 \mathrm{~min}$ vs. $120 \mathrm{~min}$ ), a reduced toxicity towards normal fibroblast cells (3T3), but an enhanced targeting ability towards human breast cancer (MCF-7). Liu et al. encapsulated atorvastatin loaded porous silicon (PSi) NPs with a reactive oxygen species (ROS)-responsive polymer, 4-(hydroxymethyl)-phenylboronic acid pinacol ester conjugated oxidized dextran (POD) and further applied the nanosystem for diabetic wound healing. ${ }^{[81]}$ The major obstacle for porous materials, burst payload release, can be overcome by the polymeric shell formation and the release kinetics can be feasibly tailored by the choice of the shell material, as the release of atorvastatin can only be triggered with the co-existence of overproduced ROS, and the release rate can be sustained for over $24 \mathrm{~h}$, making the core materials more suitable for envisioned biomedical applications.

This "shell formation induced surface stabilization" concept may be further applied to control drug loading and release if the "core" is directly composed of drug nanocrystals. Recently, Liu et al. developed a microfluidics platform with two-consecutive precipitation processes, where the first-step precipitation produced drug nanocrystals dispersing in fully dissolved polymer solution (hypromellose acetate succinate, HF) by mixing drug acetone solution $\left(10 \mathrm{mg} \mathrm{mL}^{-1}\right)$ containing HF (10 $\left.\mathrm{mg} \mathrm{mL}^{-1}\right)$ with basic aqueous solution, whereas HF sequentially precipitated 


\section{WILEY-VCH}

out by further mixing with acidic aqueous solution ( $\mathrm{pH} 3$ ), and thus, coating the drug nanocrystals in the second stage to form core/shell structures (Figure 5c). ${ }^{[44]}$ The instability issue of drug nanocrystals was overcome by the superfast coating of the polymer, as a result of the high mixing efficiency at the Re of 1300. Ultra-high flow speed further achieved considerable throughput production rate ( $\sim 700$ g per day on a single device) of the NPs. More importantly, the application of this platform increased the paclitaxel (PTX) loading degree from $\sim 6.7 \%$ to $42.6 \%$ and sorafenib (SFN) loading degree from $6.2 \%$ to $45.2 \%$, hence further potentiating the industrialization of nanomedicines.

The choices for shell materials can vary from polymers, to inorganic materials and lipids. Recently, biomimetic cell membrane-coated NPs draw increasing attention due to their superior biochemical properties, including a reduction of the mononuclear phagocyte system uptake and vaccine adjuvants-like function. ${ }^{[95]}$ Conventional methods, such as ultrasonic treatment and mechanical extrusion, may face obstacles like the inactivation of membrane proteins, destruction of NPs cores and the loss of NPs during the extrusion. Rao et al. demonstrated a microfluidic electroporation method to encapsulate $\mathrm{Fe}_{3} \mathrm{O}_{4}$ NPs using red blood cell (RBC) vesicles (Figure 5d). ${ }^{[91]}$ Electroporation, defined as a technique applying electric pulses of intensity in kilovolts per centimeter and of duration in microseconds to milliseconds, may cause a temporary loss of the semi-permeability of cell membranes. This technique was integrated with microfluidics chips to generate hydrophilic pores in the RBC's membrane. ${ }^{[96]} \mathrm{RBC}$ vesicles were harvested and purified to obtain a size of around $200 \mathrm{~nm} . \mathrm{Fe}_{3} \mathrm{O}_{4} \mathrm{NPs}(\sim 80 \mathrm{~nm})$ and $\mathrm{RBC}$ vesicles were separately injected into two inlets, after converging at the Y-shaped channel, and sequential mixing through an S-shaped channel. The mixture of the two components flew through an electroporation zone and one outlet. The electric pulses effectively promoted the entry of $\mathrm{Fe}_{3} \mathrm{O}_{4}$ NPs into $\mathrm{RBC}$ vesicles. By adjusting the pulse voltage, duration, 


\section{WILEY-VCH}

and flow velocity (50 V, $200 \mu \mathrm{s}$, and $20 \mu \mathrm{L} \mathrm{min} \mathrm{m}^{-1}$, respectively), one can achieve a complete coverage of the RBC's membrane on the $\mathrm{Fe}_{3} \mathrm{O}_{4}$ NPs without inducing the electric field caused NPs aggregation. ${ }^{[96]}$

In addition to form single core/shell structures, achieving a multi-hierarchical, multi-functional nanosystem, simultaneous co-encapsulation of two or more types of NPs with altered physiochemical properties within one matrix, is also in great demand. Liu et al. demonstrated the simultaneous co-encapsulation of PSi NPs $(\sim 200 \mathrm{~nm})$ and gold $(\mathrm{Au})$ nanoparticles $(<5 \mathrm{~nm})$ into a single polymeric matrix, and further applied it for acute liver failure theranostic therapy (Figure 5e). ${ }^{[80]}$ The application of PSi NPs enhanced the loading degree of a hydrophobic drug (2,4-((5,10-Dimethyl-6-oxo-6,10-dihydro-5H-pyrimido[5,4-b] thieno[3,2-e][1,4]diazepin-2yl)amino)benzenesulfonamide) from $0.04 \%$ to $7.8 \%$, and co-residence of Au NPs endowed the system with the capability to function as a contrasting agent for computed tomography $(\mathrm{CT})$ imaging. Moreover, the contradiction of designing Au NPs for CT imaging, namely, too small Au NPs $(<5 \mathrm{~nm})$ suffer from low plaque accumulation, whereas large Au NPs showed higher cellular toxicity, which was solved by the robust encapsulation process. It should be noted that the successful co-encapsulation of both PSi NPs and Au NPs was dependent on tailoring the surface properties of the NPs, as the co-residence of both NPs (PSi NPs and Au NPs) in a single polymeric matrix can only be achieved by separately modifying their surface with Spermine-AcDEX, which is a derivative of the shell polymer AcDEX. This further introduced the subjects for propose mechanical explanations to describe and predict the synthesis of core/shell nanohybrids using microfluidics. 


\section{WILEY-VCH}

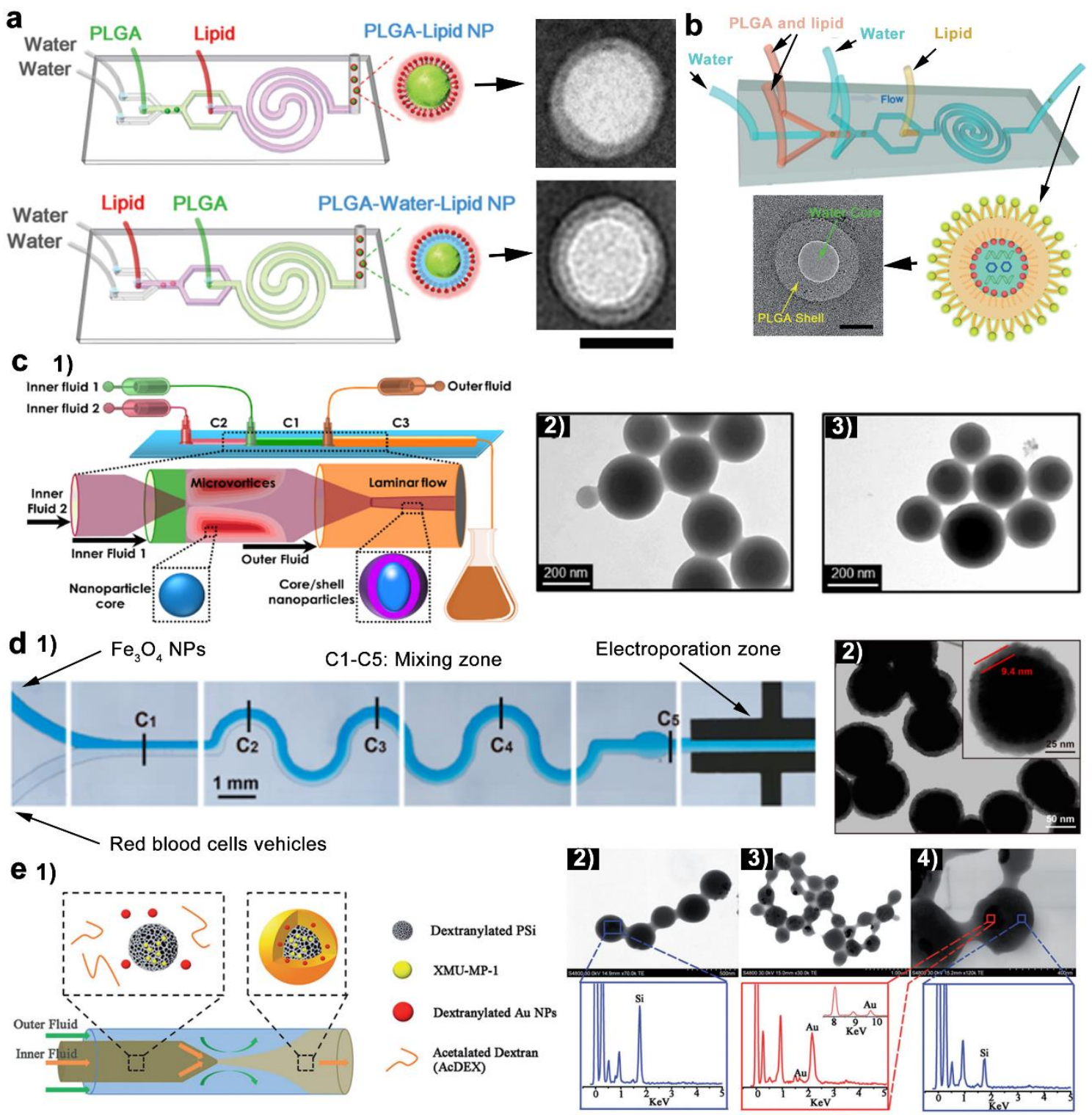

Figure 5. (a-c) Microfluidic-assisted sequential nano-encapsulation for continuously production of core/shell NPs. By altering the precipitation sequence, one can feasibly produce (a) PLGA NPs coated with lipid singe layer (up pannel), PLGA NPs coated with liposomes (down panel) or (b) Water@Lipid single layer@PLGA@Lipid single layer structures. (c1) Similar strategy can also be applied to encapsulate drug nanocrystals within enteric polymers to stabilize the drug nanocrystal, as well as the control the drug release behavior, which are confirmed by the TEM images of the produced (c2) PTX@hypromellose acetate succinate (HF) NPs or (c3) SFN@HF NPs. (d-e) Microfluidic-assisted nano-encapsulation of pre-existed NPs. 


\section{WILEY-VCH}

(d1) Application of electroporation-integrated microfluidic chip to produce RBC-vesicle encapsulated $\mathrm{Fe}_{3} \mathrm{O}_{4}$ NPs, and the TEM images of correspondingly produced nanohybrids (d2). (e1) Schematic presentation of microfluidic-assisted co-encapsulation of different NPs with different sizes (PSi NPs $\sim 150 \mathrm{~nm}$ and Au NPs $\sim 5 \mathrm{~nm}$ ). (e2) The established method solely encapsulated one type of NPs or (e3-4) simultaneously encapsulated both type of NPs. Figures

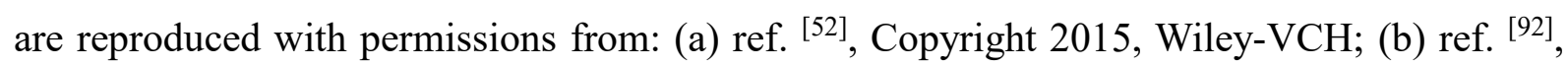
Copyright 2015, Wiley-VCH; (c) ref. ${ }^{[44]}$, Copyright 2017, American Chemical Society; (d) ref. ${ }^{\text {[77] }}$, Copyright 2017, American Chemical Society; and (e) ref. ${ }^{[80]}$, Copyright 2017, Wiley-VCH.

\subsubsection{Recent progress in describing the microfluidic-produced core/shell NPs}

The mechanisms behind the formation of core/shell structures has been less investigated. One of the first trials with potential mechanical explanations was proposed by Karnik et al. ${ }^{[76]}$ They tried to fabricate lipid/polymer nanohybrids with PLGA NPs core and lipid outer layers by using a microfluidic-assisted single-step encapsulation. PLGA in acetonitrile was used as the inner phase and lipid in ethanol/water was applied as the outer phase. The interesting phenomenon was, while keeping synthesizing parameters constant, that the size of the newly formed nanohybrid became smaller than both bare PLGA and bare liposomes. The phenomenon, as the authors hypothesized, was due to halting of PLGA NPs growth, which was caused by the lipid coverage at the hydrophobic surface of PLGA cores. Furthermore, and the pre-condition for the efficient lipid coating was the dispersion of lipid molecules within the mixing medium, which should be on the same time-order than the PLGA nucleation. A corresponding mathematical simulation and calculation were introduced to test the hypothesis, and the results showed that the timescale for total homogenization of the medium was around $0.23 \mathrm{~ms}$. This was at least an 


\section{WILEY-VCH}

order of magnitude faster than the timescale for PLGA nucleation, suggesting the PLGA nucleation was initiated under a homogenized condition. In addition, the timescales of diffusion and binding between lipid and PLGA nucleates were on the same order as the PLGA nucleation, indicating the simultaneous lipid coating at the PLGA surface.

Mathematical models have been developed to study and predict microfluidic-assisted core/shell structure formation. Gindy et al. applied multi-inlet vortex mixer (MIVM) to encapsulate colloidal Au NPs with a co-polymer (polyethylene glycol-caprolactone, PEG-PCL). ${ }^{[97]}$ Since the final size of the yielded nanohybrids was positively correlated to the Au NPs concentration, the authors deduced that the encapsulation process could be described as a diffusional growth, where the Au NPs were treated as monomers and the final formation of nanohybrids were regarded as a process of coalescence (merging of particles), and followed by the monomers association induced size narrowing effect. Therefore, based on the colloid self-assembly in the diffusion-limited regime, the authors suggested a model, described in Eq. (7): ${ }^{[97]}$

$$
\left[P_{N}\right]=[P]_{0}^{\mathrm{tot}}\left(\frac{t}{\tau}\right)^{N-1}\left(1+\frac{t}{\tau}\right)^{-N-1}
$$

where, $\left[P_{N}\right]$ is the concentration of particles composed of N Au NPs, $[P]_{0}^{\text {tot }}$ is the monomer concentration at $\mathrm{t}=0$, and $\tau$ is given in (Eq. (8)): $:^{[97]}$

$$
\tau=\frac{3 \mu}{4[P]_{0}^{\text {tot }} k_{B} T}
$$

where, $\mathrm{k}_{\mathrm{B}}$ is the Boltzmann constant, $\mathrm{T}$ is the solution temperature, and $\mu$ is the solvent viscosity. At a fixed value of $\mathrm{t}$ and $\left[\mathrm{P}_{0}\right]$ (initial number of monomers in solution), the aggregate distribution $\mathrm{N} \times\left[\mathrm{P}_{\mathrm{N}}\right] /\left[\mathrm{P}_{0}\right]$ can be represented by a function of the number of monomers $\mathrm{N}$ in aggregate $\left[\mathrm{P}_{\mathrm{N}}\right]$ (Figure 6a). Due to the ultra-high flow speed (with Re 1600), the mixing 


\section{WILEY-VCH}

timescale of multi-inlet vortex mixer (MIVM) is $3 \mathrm{~ms}$, and the approximated nucleation time of PEG-PCL, on the other hand, is $37 \mathrm{~ms}$. Based on Eq. 7, the average number of Au NPs within the nanohybrids at $\mathrm{t}=40 \mathrm{~ms}$ can be calculated. Assuming the radius of each Au NPs monomer is $4 \mathrm{~nm}$ and the packing of the monomers within the nanohybrids is randomly close-packed with a volume fraction of 0.63 , the size of the final yielded nanohybrids, as determined by the encapsulated number of monomers, can also be calculated. Experimental data were compared with calculated cluster diameters as a function of monomer concentration with sufficient correlation, and the predicted number of Au NPs within the polymeric matrix was also supported by the TEM images.

More recently, Pagels et al. further expanded the model for $\tau$ described in Eq. 8 to be applied into a wider criteria. ${ }^{[98]}$ The authors suggested a model, described in Eq. (9), to describe the core/shell formation, where the starting materials for composing the core are much smaller than the final core/shell nanohybrids. This can be observed during the polymeric encapsulation (shell) processes of hydrophobic drugs or hydrophobic polymers (core). In the study, both hydrophobic small molecules (vitamin E) and polymers (polylactide, PLA; polystyrene, PS) were successfully encapsulated by either PLA-PEG or PS-PEG. The experimental results revealed that the final size of the produced core/shell NPs was independent of the molecular weight of the core materials, which is one of the key feature for Smoluchowski diffusion-limited growth kinetics (Figure 6b). ${ }^{[99]}$ As such, derived from the Smoluchowski growth kinetics, Eq. (9) can be used to predict the size of the yielded core/shell NPs:

$$
R=\left[K\left(\frac{k_{B} T c_{c o r e} 5 / 3}{\pi \mu \rho c_{B C P}}\right)\right]^{1 / 3}
$$

where, $\mathrm{R}$ is the predicted average aggregate radius for the final nanohybrids, $\mathrm{T}$ is the absolute 


\section{WILEY-VCH}

temperature, $\mathrm{k}_{\mathrm{B}}$ is the Boltzmann constant, $\mu$ is the solvent viscosity, $\rho$ is the bulk density of the core, and $\mathrm{c}_{\mathrm{core}}$ is the mass concentration of the core material during particle assembly (one-half of the concentration at the beginning), $\mathrm{c}_{\mathrm{BCP}}$ is the mass concentration of the shell material during particle assembly (one-half of the concentration at the beginning), and $\mathrm{K}$ is a single scaling constant with the value $253 \mathrm{~ms} \cdot \mathrm{g}^{1 / 3} \mathrm{~m}^{-1}$. The main goal of Eq. (9) is to provide a mean for the size and drug loading optimization with a given hydrophobic compound. However, it should be noted that the key parameter within the Eq. (9), K, was summarized from the actual experimental data and the successful prediction may likely be dependent on the stabilizing hydrophilic polymer-block used. Nonetheless, considering the most commonly used stabilizer within this study (PEG), the simplicity of Eq. (9) remains a relevant model that can be easily applied to producenanohybrids.

To understand the process to encapsulate the core NPs, Hasani-Sadrabadi et al. applied molecular dynamics (MD) simulations to delineate the paclitaxel loaded chitosan core/Eudragit shell formation process. ${ }^{[94]}$ A T-shaped microfluidics chip was coupled with a Tesla-shaped chip to produce core (chitosan)/shell (Eudragit FS 30D, poly(methyl acrylate-co-methyl methacrylate-co-methacrylic acid)) NPs. A 3D cell consisting of Eudragit and chitosan chains was fabricated by use of COMPASS (condensed-phase optimized molecular potentials for atomistic simulation studies) force field. After 600 picoseconds NVT (moles (N), volume (V), and temperature (T) are conserved for the canonical ensemble) MD simulations, the distance between Eudragit and chitosan layers in the equilibrated cell became closer, which was further corroborated by the binding energy, and the strong intermolecular hydrogen bonding interactions between Eudragit and chitosan, as such, provided further insights for interpreting the mechanism of successful encapsulation (Figure 6c). 


\section{WILEY-VCH}
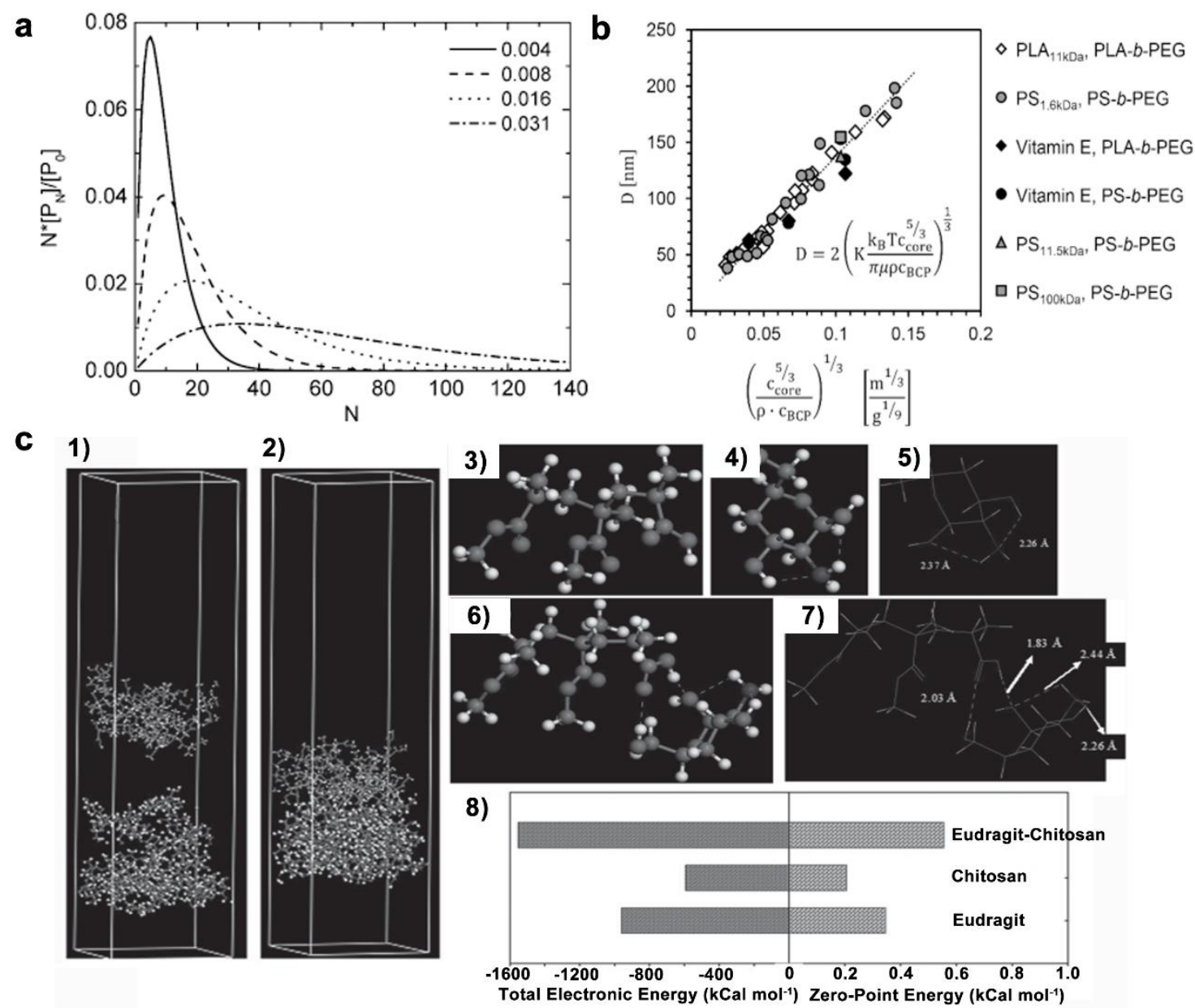

Figure 6. (a) Based on the diffusion-limited colloid self-assembly model, a plot of aggregate distribution as a function of the number of Au cluster monomers in the aggregate is generated to predict the size of yielded nanohybrids at the fixed time of $40 \mathrm{~ms}$. (b) The actual size of produced core/shell nanohybrids compared with the predicted size for a given formulation calculated from Eq. (9) (dash line), with a value of $\mathrm{K}=253 \mathrm{~ms} \cdot \mathrm{g}^{1 / 3} \cdot \mathrm{m}^{-1}$. (c) MD simulation was applied to study the interaction between Eduragit and chitosan. (c1) Initial structure and (c2) after 600 ps MD simulations. Optimized model structure of (c3) Eudragit; (c4-5) chitosan; (c6) Eudragit-chitosan cluster and (c7) hydrogen bonding between Eudragit and chitosan. (c8) Quantitative total electronic energy and zero-point energy of Eudragit, chitosan and Eudragitchitosan cluster. Figures are reproduced with permissions from: (a) ref. ${ }^{\text {[97] }}$, Copyright 2008, American Chemical Society; (b) ref. ${ }^{[98]}$, Copyright 2018, American Chemical Society; and (c) 
ref. ${ }^{[94]}$, Copyright 2016, Wiley-VCH.

\section{Microfluidic production of microparticles/droplets}

The aforementioned methods to prepare NPs usually apply single phase flow within the fluidic channel, i.e., the multiple fluids within the channel are miscible with each other. And in the following section, we will focus on another fundamental flow regime for microfluidics: multiphase flow, which is composed with two or more immiscible phases, more specifically, immiscible liquid/liquid systems.

The prevailing applications for multi-phase flow based microfluidic devices are micro-particles or droplets production, despite there are also nano-emulsions feasibly fabricated via microfluidics. ${ }^{[100]}$ The challenges for producing nano-droplets or emulsions come from the flow dynamics within the microfluidics channel and the mechanisms of droplet production. For nanoemulsion, the free energy of the droplets is higher than the free energy of the separate phases, which means that nanoemulsion is thermodynamically unstable. And the preparation process usually requires a strong energy input. Based on the equation (Eq. 10) proposed by Gupta et al., ${ }^{[101]}$

$$
\varepsilon=\frac{c \mu_{d}^{0.8} \sigma^{2}}{\rho_{d}{ }^{0.5} \rho_{c} \mu_{c} d^{0.5}}
$$

where $\varepsilon$ is the input energy intensity, $\mu_{\mathrm{c}}$ and $\mu_{\mathrm{d}}$ separately represent for the viscosity of continuous and dispersed phase, $\rho_{\mathrm{c}}$ and $\rho_{\mathrm{d}}$ separately represent for the density of continuous and dispersed phase, $\sigma$ is the interfacial tension, $\mathrm{c}$ is a constant and $\mathrm{d}$ is the diameter of the emulsion. For a typical $\mathrm{O} / \mathrm{W}$ emulsion with the size $100 \mathrm{~nm}, \varepsilon$ is on the order of $10^{8}-10^{10} \mathrm{~W} \mathrm{~kg}^{-1}$, which set an obstacle for conventional microfluidics production, and the increased size of produced 


\section{WILEY-VCH}

emulsions can significantly reduce the necessary energy input, as such more suitable for conventional microfluidics production.

\subsection{Microfluidics production of microparticles: advantages over conventional methods}

The adaptation of microfluidics to the production of microsized systems has become more popular since the first research work made more than 10 years ago. ${ }^{[102]}$ The main reasons of the adoption of this technology stem from the homogeneity of the emulsions and the high degree of control over the process. ${ }^{[103-105]}$ The control over the whole production process is possible due to the properties of the fluids in the microfluidic channels.

The conventional production of microemulsion and microparticles is based either on high- or low-energy methods. High energy methods (e.g. high shear homogenizers and sonication) form emulsions by introducing high shear forces to disrupt the phases, leading to their mixing. ${ }^{[106]}$ The formation of one emulsion is not a spontaneous phenomenon: it requires energy contribution to compensate the energy loss associated with an increase in the surface area. ${ }^{[107]}$ The energy required to expand the surface can be described according to Eq. (11):

$$
\Delta G=\Delta A \gamma-T \Delta S
$$

where, the free energy of formation $(\Delta \mathrm{G})$ is assumed positive due to the positive contribution of the energy to increase the interfacial area $(\Delta \mathrm{A} \gamma)$, given by the increase in the area $(\Delta \mathrm{A})$ multiplied by tension at the interface $(\gamma)$, with a minimal contribution from the entropy, resulting from the dispersion.

The dimension of the emulsion droplets produced in bulk is regulated by the interplay of two different processes: droplets breakdown and droplets coalescence. ${ }^{[108]}$ The breaking down of the droplet happens if the shear applied by the instrument is bigger than the Laplace pressure of the complex mix. In particular, the Laplace pressure $\mathrm{p}$ (difference in the pressure between 


\section{WILEY-VCH}

the inside and outside of the emulsion droplets) for a spherical droplets is described, according to Eq. (12):

$$
p=2 \gamma R
$$

where, the pressure p equals the radius of the spherical droplet multiplied by interfacial tension. Eq. (12) justifies the need for high energy required to deform, and eventually break-up the spherical dropswhich is reflected in higher Laplace pressure, and thereby, higher energy needed. An important descriptor of deformation in the droplets is the Weber number (We), defined by Eq. (13):

$$
W e=G \eta r 2 \gamma
$$

where, the We is correlated with the ratio of the external shear given to the system $(\mathrm{G}$ is the velocity gradient and $\eta$ is the viscosity) on the Laplace pressure $(2 \gamma r)$. This number increases with the augment in the deformation, thereby higher energy is needed to produce smaller emulsions. ${ }^{[107]}$ Taylor's Eq. (14) allows precise estimation of the size of the droplets when the viscosity of the continuous phase is negligible and the system contains a low percentage of the dispersed phase:

$$
r \propto \frac{\gamma}{\eta \gamma^{\prime}}
$$

where, the size (radius, $r$ ) is proportional to the ratio between the interfacial tension $(\gamma)$ divided by the viscosity of the continuous phase $(\eta)$ multiplied by the shear rate $\left(\gamma^{\prime}\right)$. Taylor's equation helps understand the role played by the surfactant in the process: the role of a surfactant is to decrease the surface tension $(\gamma)$, which is directly proportional to the radius of the droplet. However, the fundamental function of a surfactant within an emulsion system is the 


\section{WILEY-VCH}

stabilization of the emulsion itself, given by the positioning of the surfactant molecules at the interface between droplets and continuous fluid, which can effectively prevent the coalescence of the droplets. ${ }^{[108]}$

Different theories have been proposed to describe the mechanisms behind this observed phenomenon, namely a stabilization due to the Gibbs-Marangoni's effect in the early stages of emulsification, electrical repulsion, solvation barrier, viscosity of the surfactant solution, and hydrodynamic barrier to the flow of continuous fluid in the thin layer between two emulsion droplets. However, the general mechanism of stabilization is mediated by the balance between the reduction in the interfacial area derived from the merging of two droplets with the interfacial tension constant. In presence of a surfactant, the interfacial tension varies if the rate of the coalescence between the two droplets is faster than the rate of desorption of the surfactant from the interface, which results into an imbalance in the equilibrium of the adsorption. This leads to an increase in the chemical potential of the surfactant that can be alleviated only by either desorption of the surfactant molecules from the interface or from an increase in the interfacial area, counterbalancing the tendency to coalesce. ${ }^{[109]}$

For single-phase flow facilitated NPs production, as discussed in section 2, the chip geometry is majorly composed by co-flow, whereas multiple geometries have been designed for microparticles production, and the mechanisms of droplet formation in microfluidics are generally dependent on the type of chip, its characteristics, and the attributes of the fluids. The different chip geometries exploited in the development of droplet-based emulsions and microparticles have been reviewed elsewhere. ${ }^{[72,103]}$ Briefly, as presented in Figure 7, the most commonly employed geometries in 2D are T- and Y-junctions, while for the 3D design, flow 


\section{WILEY-VCH}

focusing, co-flow, and different combinations of the previous for the production of double emulsions. ${ }^{[104]}$
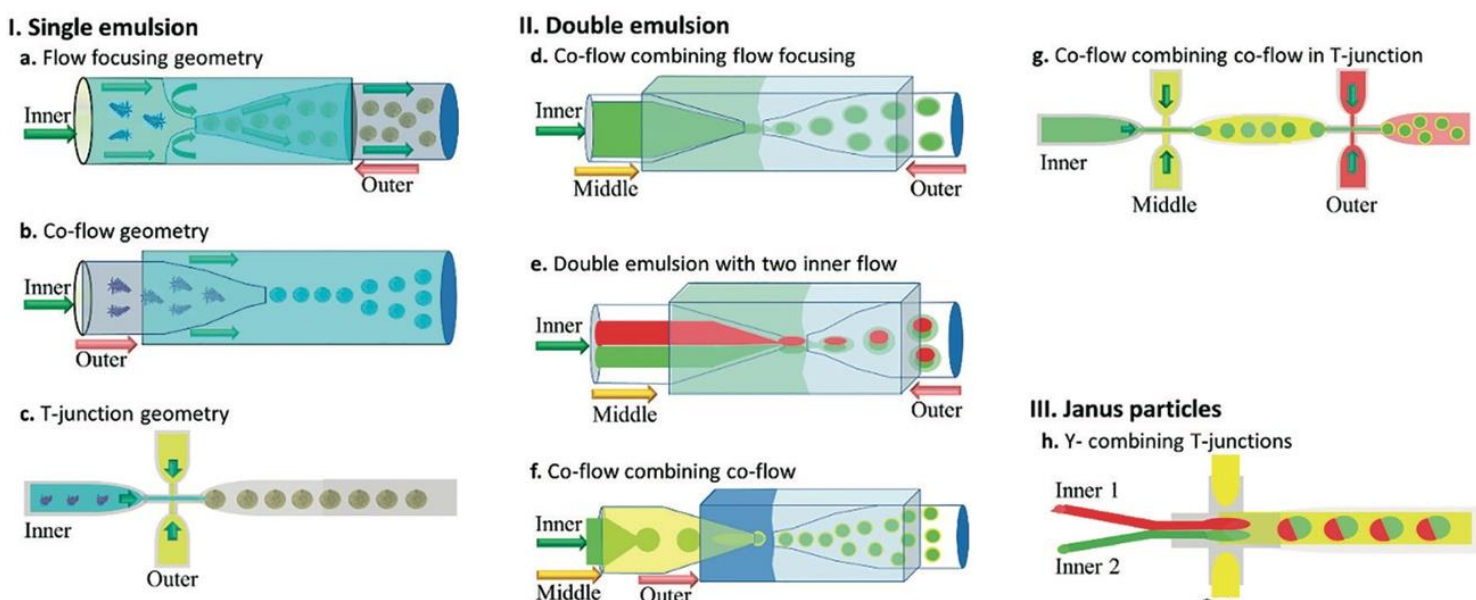

III. Janus particles

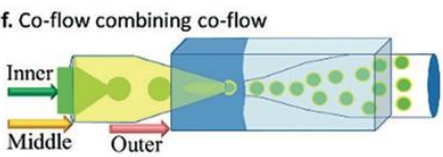

h. Y- combining T-junctions

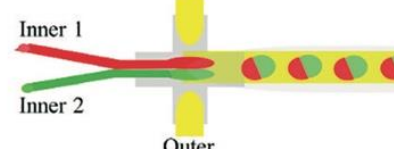

Figure 7. Most commonly employed device configurations in droplet-based microfluidics. (a-c) Single emulsion with flow focusing and co-flow (a,b) applicable to both glass capillary and 2D chip, while T-junction (c) is proper only of 2D PDMS devices. (b-g) Double emulsion configurations: as for glass capillary-based devices, combinations of co-flow with flow focusing, co-flow followed by co-flow, or multiple inner flow are reported (d-f), while for 2D devices, geometries combining co-flow with T-junctions or Y-junction combined with $\mathrm{T}$ junction. (h) Microfluidic channel configuration for producing Janus particles. Reproduced with permission from ref. ${ }^{[103]}$, Copyright 2017, Royal Society of Chemistry.

The main parameters having an effect on the fluids behavior have been presented above and are the Peclet number, Re, and capillary number $(\mathrm{Ca})$. Ca describes the ratio between the viscous forces of the fluid over the surface tension, according to Eq. (15):

$$
C a=\frac{\eta v}{\gamma}=\frac{v \rho v}{\gamma}
$$




\section{WILEY-VCH}

where, $\eta$ is the dynamic viscosity of the fluid, $v$ is the velocity of the fluid, and $\gamma$ is the surface tension. ${ }^{[110]}$ The dynamic viscosity is given by the static viscosity, $\rho$, multiplied by the velocity of the fluid.

The capillary number is of particular importance in droplet-based microfluidics. because it allows the investigation of different break-up patterns described by different ranges of capillary numbers. ${ }^{[111]}$ In the case of low values of the capillary number (range $<10^{-2}$ ), the formation of the droplet is not influenced by the shear stress and it is only dependent on the accumulated pressure in the inner channel to form a thread and ultimately squeeze out the droplet. ${ }^{[112]}$ The droplet formation will occur when the maximum extension of the droplet is higher than 1 , according to Rayleigh-Plateau instability. ${ }^{[113]}$ A useful implementation of the capillary number (extensional flow capillary number) can help distinguish between breaking and non-breaking parameters. To allow this, the radius of the droplet needs to be included amongst the parameters on the numerator of the Ca number fraction. ${ }^{[113]}$ However, according to other simulations, the droplet formation has been shown to be independent from the capillary number and to rely on the geometrical characteristic of the device, viscosity of the oil phase and the contact angle between the two phases. ${ }^{[114]}$ This alternative model accurately describes the droplet formation at low flow rates. Furthermore, the first and the second model can be integrated considering the extensional flow capillary number, defined in Eq. (16): ${ }^{[113]}$

$$
C_{G}=C a / w_{0}
$$

where, $\mathrm{C}_{\mathrm{G}}$ is the geometrical parameter and $\mathrm{w}_{0}$ is the width of the channel.

In the case of class capillary microfluidics, the droplet formation is achieved in two different regimens, dripping or jetting. ${ }^{[104]}$ The dripping regimen represents absolute instability, meaning 


\section{WILEY-VCH}

that the forces influencing the perturbation of the fluid are fixed, resulting in a system-intrinsic frequency. On the contrary, in jetting conditions, the perturbations are amplified throughout the fluid, leading to higher polydispersity of the droplets. ${ }^{[115]}$ By carefully adapting the parameters (increasing the inner flow rate until a threshold where the inertial forces are higher than the surface tension) the propagation of the oscillations occurs in a capillary wave fashion, leading to the pinching of the droplet. ${ }^{[15]}$ The careful optimization of the inner and outer fluid velocity allows the production of homogenously dispersed droplets even in jetting regimen. ${ }^{[15]}$

\subsection{Moving from colorful droplets to artificial cells and bioreactors}

The initial and one of the most important applications of microfluidics produced microparticles or droplets are encapsulating bioactive compounds, including small molecule drugs, peptides/proteins and DNA/RNA. Several reviews have been proposed previously to summarize microfluidic fabrication of microparticles for drug loading/delivery applications. ${ }^{[116]}$ Yet, inspired by the recent evolving molecular biological concepts and techniques, microfluidic-produced micro-particles and droplets may also become an alternative platform for multiple applications.

The first proof-of-concepts in droplet-based microfluidics have been focusing on the production of complex emulsions, visualized with the use of hydrophilic and lipophilic colors (Figure 8). Complex architectures have been created in glass capillary microfluidics by a careful engineering of the geometry of the capillary, to introduce elements in series or in parallel, and thereby, producing multiple smaller droplets within a bigger one. 


\section{WILEY-VCH}

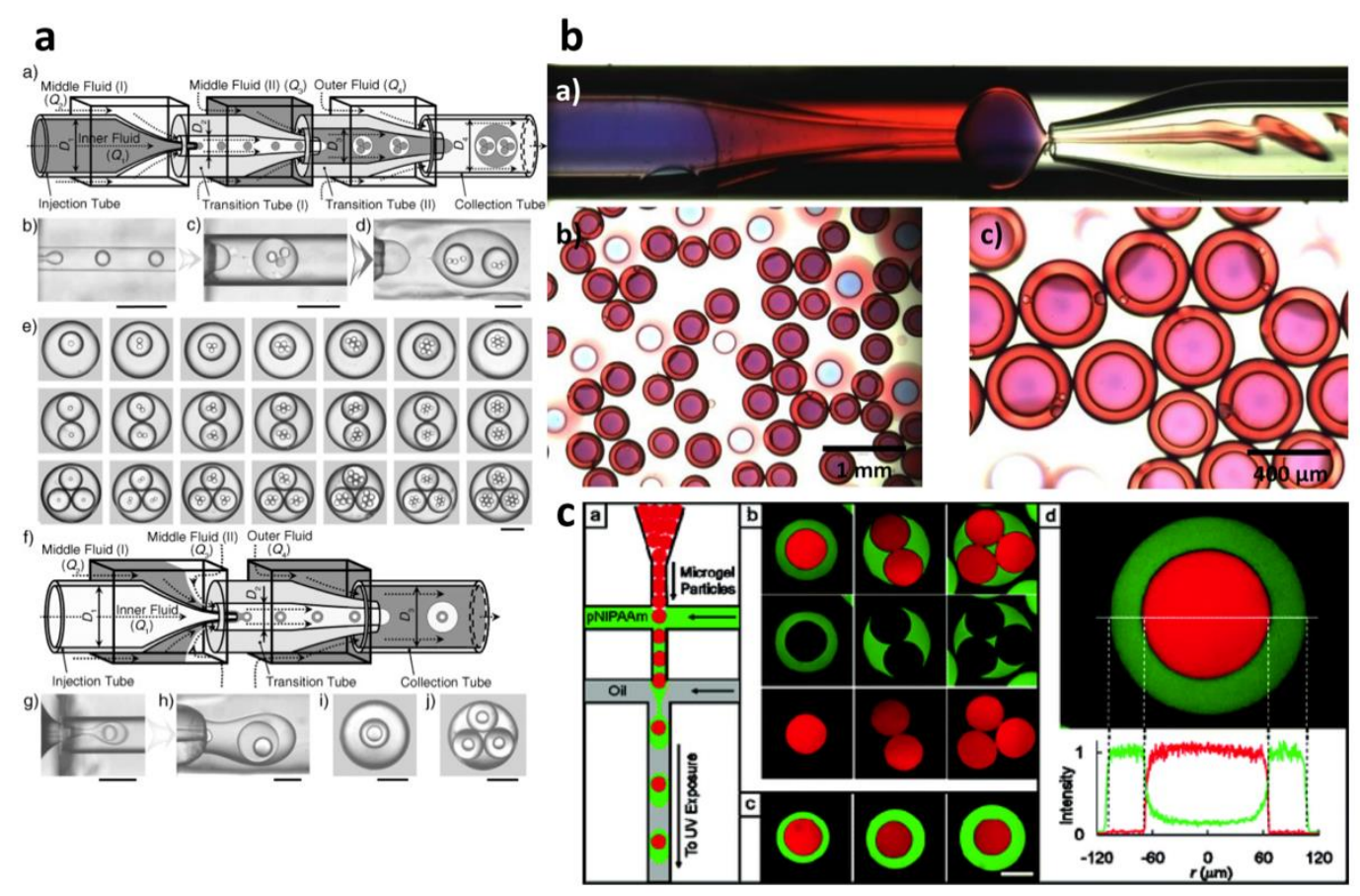

Figure 8. (a) Device geometries and process for the generation of triple emulsions. a. Schematic of the device with the different elements connected in series; $b-d$. Micrographs of the different phases of the emulsification process (stage I to stage III); e. Micrographs of the produced emulsions, displaying the high control in the number of droplets added in each of the stages; $\mathrm{f}$. Alternative configuration of the device eliminating the need for the middle fluid compartment; $\mathrm{g}-\mathrm{j}$. Microphotographs of the improved process. (b) Double emulsion proof-of-concept of an innovative platform for the chip assembly. a. micrograph of the assembled device, running in a flow focusing configuration; b. and c. micrographs of the obtained emulsions. (c) Production of multiple layers of polymers in a $2 \mathrm{D}$ device with a double $\mathrm{T}$-junction configuration. a. Schematic of the device with the elements in series; b. and c. Micrographs of the obtained gels, highlighting the effect of the different parameters on the shell thickness. d. Spatially-resolved profiles of the fluorochromes to investigate the interpenetration between the two polymers. Figures are reproduced with the permission from: (a) ref. ${ }^{[117]}$, Copyright 2007, WILEY-VCH; 


\section{WILEY-VCH}

(b) ref. ${ }^{[118]}$, Copyright 2016, Elsevier; (c) ref. ${ }^{[119]}$, Copyright 2010, American Chemical Society.

These proof-of-concepts have been of the utmost importance in the exploration of the ranges of parameters producing complex systems, facilitating their further development. The engineering of devices like the one presented in Figure 8a enables the control over every single module in terms of frequency of droplets produced in each module. The range of capillary numbers has been selected to achieve the droplet break-up under conditions of absolute instability, or dripping, allowing to predict the size of the droplet by calibrating the flow rate of the outer fluid. ${ }^{[117]}$ Moreover, the authors reported empirical relationships between the diameter of each layer of droplets and the flow rate that resulted into a control over the desired number of droplets within each of the layers by simply modifying the flow rate, according to Eq. (17):

$$
N_{1}=\frac{f_{1}}{f_{2}}=\frac{Q_{1} /\left(\pi d_{1}^{3} / 6\right)}{\left(Q_{1}+Q_{2} / \pi d_{2}^{3} / 6\right)}=\frac{Q_{1}}{Q_{1}+Q_{2}} \frac{d_{2}^{3}}{d_{1}^{3}}
$$

where, the number of inner droplets $\left(\mathrm{N}_{1}\right)$ is predicted by the control over the flow rates of the different modules ( $f_{1}$ and $f_{2}$ being the formation rates of the inner and outer droplets, $Q_{1}$ and $\mathrm{Q}_{2}$, the flow rates of inner and outer fluid, respectively, and $d$ the diameters of inner of outer droplets). ${ }^{[117]}$ As evident from Eq. (17), a careful adjustment of the flow rates between the different modules is governing the type of complex structure obtained (Figure 8a).

The proof-of-concept has been employing rather straightforward to inner and outer solutions like poly(dimethylsiloxane) oil, octanol, glycerol, kerosene, all containing 1-3\% of surfactants like Dow Corning 749 fluid (for the oil phase) and polyvinyl alcohol (PVA) for the water 


\section{WILEY-VCH}

phase. ${ }^{[117,118]}$ Additionally, colorants were used to help visualize both the flows and droplet formation within the device and the final structure of the vesicles (Figure 8b), such as trypan blue can be added to water phase, while nile red can stain oil phases. ${ }^{[118]}$

Importantly, the production of complex structures by microfluidics is not limited to glass capillary devices. Soft lithography-printed devices (like PDMS) can produce homogeneous droplets by flow focusing ${ }^{[120]}$ or geometrically-mediated breaking (T-junctions). ${ }^{[113,121]}$ As presented in Figure 8c, T-junction devices can be engineered to produce core-shell structures. ${ }^{[119]}$ Once again, as demonstrated for the glass capillary devices, the number of inner droplets within the outer shells and the thickness of the shell are controlled by the flow rates of the inner, middle and outer phases. ${ }^{[119]}$ However, these devices are less robust towards organic solvent when compared to the glass capillary ones. ${ }^{[122]}$

\subsubsection{Microvesicles: from polymerosomes to artificial cells}

The first examples of applications for the microdroplets have been in the development of vesicles or solid microparticles for drug delivery. ${ }^{[103,122]}$ Vesicles (e.g., polymerosomes and liposomes) can be defined as an enclosure of fluid surrounded by a bilayer of amphiphilic molecules. ${ }^{[122]}$ The conventional preparation methods are co-solvent method (solvent switch, where the amphiphilic molecules are dissolved in organic solvent, before gradually adding an aqueous solution), film rehydration method (organic solvent is used to dissolve the amphiphilic molecules, before being completely evaporated, creating a film of the amphiphiles, ready to be rehydrated with aqueous solutions), and direct dissolution method (amphiphiles precipitate in the water solution, followed by a self-assembly process). ${ }^{[123]}$ Each of these methods lead to a vesicle population with quite wide polydispersion, and the need for further homogenization steps. ${ }^{[123]}$ 


\section{WILEY-VCH}

However, vesicles can be easily produced by double emulsions templates in microfluidics: the amphiphilic molecules are dissolved within the organic middle phase in a water-in-oil-in-water emulsion setup. ${ }^{[122,124]}$ The optimization of the flow conditions affects the radius of the emulsion droplets, thereby the size of the final vesicles. The proof-of-concepts have led to the development using diblock copolymers (e.g., poly(n-butyl acrylate)-poly(acrylic acid) and phospholipids) in tetrahydrofuran that self-assemble at the interfaces between the water and oil interfaces (Figure 9a). ${ }^{[125,126]}$ Lipidic vesicles have also been produced via T-junction droplets breaking (Figure 9b). ${ }^{[127]}$ Importantly, the viscosity of the outer water phase helps improving the flow focusing geometry, while the volatility of the organic solvent favors the self-assembly of the amphiphiles, facilitating the formation process. ${ }^{[125,128]}$ Two different mechanisms have been proposed for the formation of the vesicles from the double emulsion: the evaporation of the organic solvent induces the formation of a depletion force, which in turn initiate the dewetting process (Figure 9c) ${ }^{[129]}$ However, the process is highly influenced by the speed of the evaporation in the final dewetting stage, which may break the vesicles. ${ }^{[126]}$ Thereby is of paramount importance to control the speed of the solvent removal. This can be controlled through a control on the concentration of the organic solvent in the outer solution that is also directly correlated with the final size of the vesicles (the longer the time allowed for the dewetting-evaporation process, the smaller the vesicles). ${ }^{[126]}$ Another way to control the size of the vesicles or the final PLGA microparticles was demonstrated by $\mathrm{Tu}$ et al. through osmotic annealing. ${ }^{[130]}$ The annealing is controlled by the concentration of a solute in the water phases (inner and outer). Thereby, to control the tension on the vesicle is by having the dewetting in concentrated glycerol solutions (80\% and above). ${ }^{[126]}$ A second mechanism proposed for the formation of the vesicles is the pinning of the amphiphiles to the inner water vesicles, allowing the translocation of the inner vesicle through the oil phase till the outer interface. ${ }^{[126]}$ 


\section{WILEY-VCH}

a

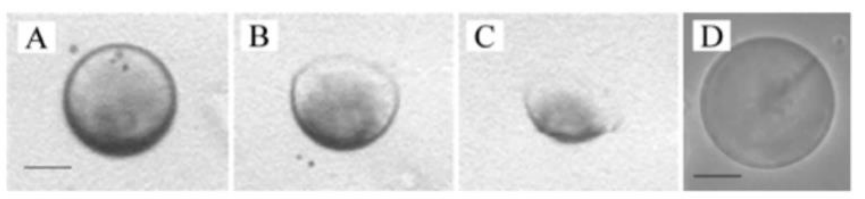

C

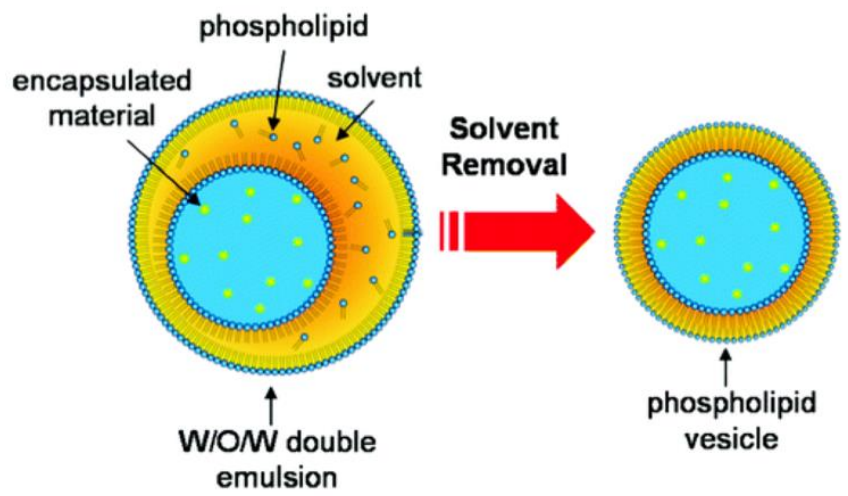

b

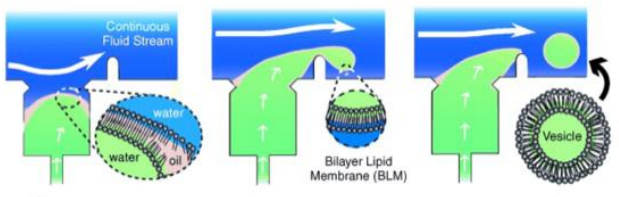

d
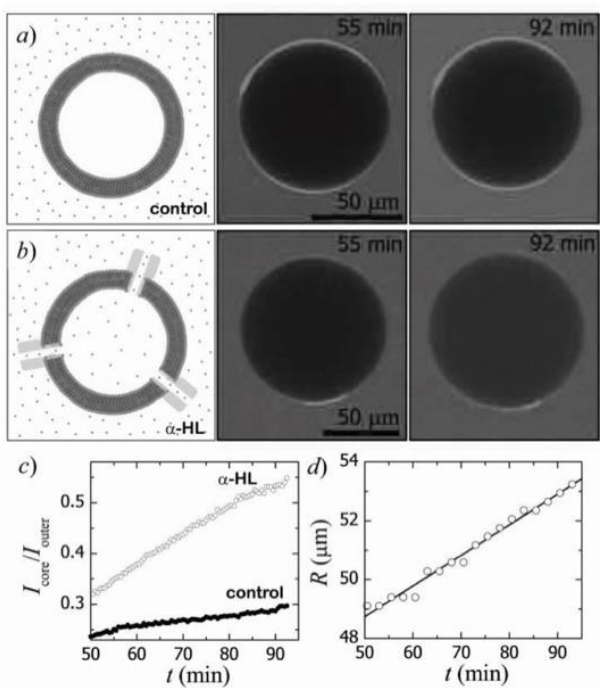

Figure 9. (a) Proof-of-concept of the dewetting of double emulsions obtaining polymerosomes.

A-C. Micrographs of the organic solvent evaporation; D. Micrograph of the final polymersome.

(b) Engineering of phospholipidic bilayer vesicles via T-junction microfluidics. Schematic of the vesicle formation process. (c) Proposed dewetting mechanism for the formation of phospholipid vesicles starting from double emulsions: during the solvent evaporation, the phospholipids dissolved in the middle organic phase reassemble at the interfaces between the oil and the water phases, creating a bilayer phospholipidic vesicle. (d) Suitability of thin-layer double emulsion technique for the production of functional models of cell membrane. The produced vesicles presented membrane heterogeneity and could accommodate a pore forming protein ( $\alpha$-hemolysin) as illustrated in the scheme and confirmed by the gradual accumulation of sulphorhodamine entering through the pores created by the protein. Figures are reproduced with the permission from (a) ref. ${ }^{[125]}$, Copyright 2005, American Chemical Society. (b) ref. 
${ }^{[127]}$, Copyright 2009, WILEY-VCH. (c) ref. ${ }^{[126]}$, Copyright 2008, Americal Chemical Society; (d) ref. ${ }^{[131]}$, Copyright 2013, WILEY-VCH.

Recently, micro-sized vesicles (mainly giant unilamellar vesicles) have surged to a new role as ideal models of artificial cells and cell membrane. ${ }^{[132]}$ The advantages derived from the use of microfluidics technique for their production reside mainly in the degree of control achievable on size, lamellarity, membrane composition, and payload. ${ }^{[131,133,134]}$ At the same time it is possible to produce high number of such vesicles, reducing the overall costs. ${ }^{[133]}{ }^{[133,135]} \mathrm{A}$ downside of the production of giant unilamellar vesicles by double emulsion in microfluidics is the lacking heterogeneity in the composition of the membranes. ${ }^{[131,136]}$ While cells and artificial membranes produced through conventional methods show high membrane heterogeneity with the presence of lipid rafts, the presence of organic solvents and the production process in microfluidics leads to the production of homogenous vesicles. A careful selection of the volatility of solvents employed in the middle organic phase results in the formation of heterogenic domains, with the possibility to insert membrane proteins, like the pore-forming $\alpha$-hemolysin (Figure 9d). ${ }^{[131]}$ Alternatively, giant unilamellar vesicles can be produced with $\mathrm{MHz}$ yield through droplet stabilization with the possibility to inject biomolecules through pico-injection. ${ }^{[137]}$ In particular, the formation of the vesicles is promoted by the injection of $\mathrm{Mg}^{2+}$ ions within the droplets, promoting the formation and the release of the vesicles. ${ }^{[137]}$ Interestingly, however, by mechanical compressing the giant unilamellar vesicles, Robinson et al. observed a spontaneous rearrangement of the lipid domains culminating into a the formation of bigger domains. ${ }^{[132]}$ 


\section{WILEY-VCH}

Artificial cells and artificial cell membranes help in the breaking down of the complex cellular machine, one mechanism at the time to better understand their function. ${ }^{[133][133,138]}$ However, the complexity of the cellular environment has proven difficult to recreate either in terms of membrane features (as discussed above) or in terms of intracellular organelles complexity. Cytoskeleton is one of the cellular features that researchers have tried to mimic; natural components of the cytoskeleton have been loaded within the vesicles, or the rigidity of the cytoskeleton has been provided by hydrogels. ${ }^{[139,140]}$
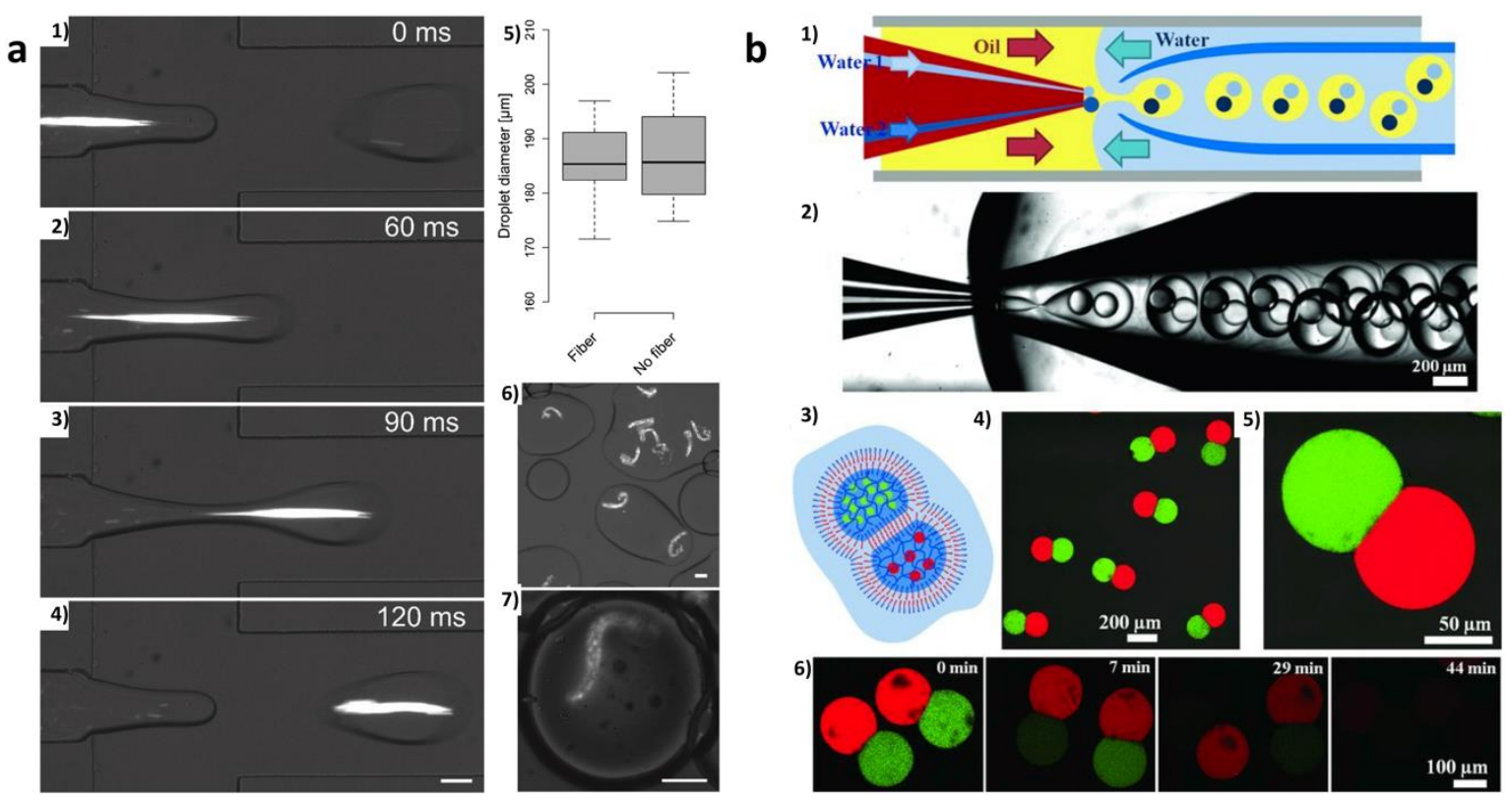

Figure 10. (a) Controlled formation of alginate microfilaments and their encapsulation within vesicles to mimic the cytoskeleton fibers. 1)-4) Micrographs of the process of fiber encapsulation within the droplets; 5) Comparison of the size distribution between empty and fiber-loaded vesicles; 6)-7) Micrographs collected at different time points, showing that the fiber maintains its shape and dimension over time, confirming the success of the gelation process. (b) Engineering of dumbbell-shaped polymerosomes, creating two compartments with different payloads. 1) Schematic of the microfluidics set up. 2) Micrograph of the 


\section{WILEY-VCH}

polymerosomes production within the chip. 3) Schematic of the structure of the dumbbell polymerosomes. 4)-5) Confocal micrographs of the dumbbell-shaped polymerosomes highlighting the two different types of hydrogels loaded (different fluorochromes). 6) Release behavior in ultrapure water over time. Figures are reproduced with the permission from: (a) ref. ${ }^{[140]}$, Copyright 2016, Royal Society of Chemistry; (b) ref. ${ }^{[141]}$, Copyright 2013, WILEY-VCH. In one interesting example, the cytoskeleton fibrils microtubules have been mimicked by alginate microfibers (Figure 10a). Both the synthesis of the microfibers and their encapsulation within the vesicles have been performed within the same device. ${ }^{[140]}$ The length of the alginate fibers can be controlled through the application of a pulsatile pressure regimen in the first sheathing buffer, effectively cutting the fiber off at the desired length. The gelation of the fiber is achieved further down the channel, with the input of a second buffer solution containing $\mathrm{Ca}^{2+}$ ions, before a single-step emulsification into the final vesicle. ${ }^{[140]}$ In the setup conditions, only one in three droplets presented a fiber encapsulated due to a difficult interfacing between the pulsatile conditions required for the cutting of the fiber and the controlled flow rate demanded for the droplet formation. Contractility movements of vesicles loaded with microtubulues and its dependency on ATP has also been shown. ${ }^{[142]}$ The intracellular elastic module can also be achieved through the use of hydrogels with different stiffnesses. ${ }^{[143]}$ However, different intracellular compartment present different stiffness together with different payload. Dumbbellshaped polymerosomes can offer a partial solution by enabling the selection of different payload and stiffness in each of the two compartments through the two parallel flows of the inner water phase ${ }^{[141]}$ Alternatively, the formation of actin filament and microtubules was achieved through picoinjections of G-actin and tubulin, together with polymerization buffers, within droplet stabilized giant unilamellar vesicles. ${ }^{[137]}$

Moving forward, a control over the cell size, shape, and encapsulation of protein systems can 


\section{WILEY-VCH}

be reliably achieved by trapping the droplets in 3D structures in PDMS systems. ${ }^{[144]}$ By applying different flow rates, modifying the dimensions of the trap, pr creating different pressures thanks to transmembrane osmotic pressure, this systems can produce bacteria-like vesicles, discoidal-like cells, and achieve an orientation-controlled alignment of protein fibers (microtubules or collagen) within the artificial cells. ${ }^{[144]}$

The proof-of-concept design of multiple droplets within the same emulsion have inspired vesosomes mimicking the organelles structure and have been investigated as bioreactors. ${ }^{[145]}$ In particular, Deng et al. have investigated different configurations of the devices to answer to different needs of the artificial cell models, including a protein-mediated self-assembly of organelles. ${ }^{[146]}$ The double emulsions vesicles have been prepared by phospholipidic bilayer, stabilized by PEG, PVA and pluronic as surfactants in the water phases. The control over the different structures achieved was obtained by a control over the flow rate of the different phases, according to the empirical Eq. (17). However the downside of their technique is the impossibility to modify the compartments after production, which may be solved by modifying picoinjection methods. ${ }^{[147]}$ In a simpler design, Ugrinic et al. developed proteinosomes, vesicles formed by proteins conjugated with poly( $\mathrm{N}$-isopropylacrylamide) to render them amphiphilic. ${ }^{[148]}$ The proteins forming the shell of the vesicles have been proven to be functional and, by loading into the vesicles proteins or enzymes constituting the following step in the signal cascade, multiple step enzymatic reactions could be performed. ${ }^{[148]}$ Finally, taking advantage from the possibility of modifying single-strand DNA into DNA origami, intracellular lipidic moieties can be inserted within the artificial cells by DNA preprogramming, as reported in detail in other reviews. ${ }^{[147]}$ 


\section{WILEY-VCH}

The future of artificial vesicles resides in the development of novel methods to improve the yield and the maturation time of the vesicles ${ }^{[135]}$ or in the encapsulation of living cells within the vesicles to serve as bionic organelles. ${ }^{[149]}$ Alternatively, microfluidic-based artificial cells can model the interaction between viruses and host cells, allowing for the high throughput screening of antiviral compounds within the picoliter volume of the vesicle. ${ }^{[150]}$ Multiple questions remain about the possibility to formulate an universal "cytoplasmatic" buffer, about the different methodologies to deliver different payloads in succession, to the construction of complex intracellular structures.

\subsubsection{Microparticles and microcapsules: from drug delivery to bioreactors}

The controlled emulsion droplets obtained in microfluidics serve also as excellent template for solid particles. ${ }^{[151]}$ Given that the starting template is still constituted of emulsions, the control over the size, the homogeneity, and the structure of the particle is mediated by the geometry of the device and the control over the flow rate of the different phases flowing within the chip. The simplest examples of solid particles are derived from single emulsion templates, with the production of a solid matrix-like particle after solvent evaporation and dewetting (Figure 11a). ${ }^{[152]}$ Such systems are formulated from polymers (e.g., PLGA) or lipid dissolved in the organic phase, with a surfactant added to the water phase to stabilize the emulsion droplets. ${ }^{[152,}$ ${ }^{153]}$ Drug, imaging agent, micro- and nano-particles are amongst the first payloads evaluated within possible biomedical applications as therapy or imaging agent (Figure 11b). ${ }^{[103,154]}$ More complex structures, including core-shell, capsules, porous particles have been engineered from double emulsions (Figure 11c) ${ }^{[104,155,156]}$ The development of such systems has been mainly 


\section{WILEY-VCH}

focused on the release profile of the payload from the particle, important for drug delivery applications. ${ }^{[103]}$

a

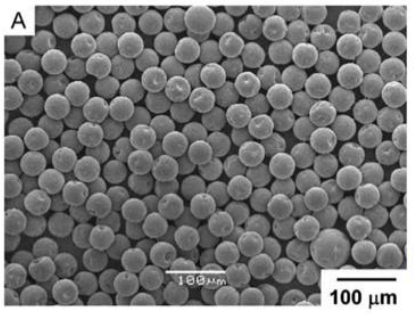

B

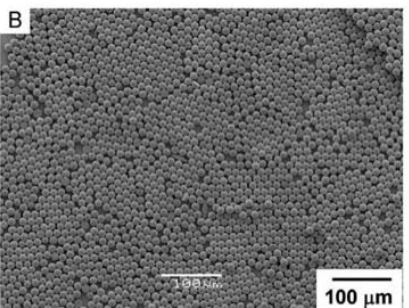

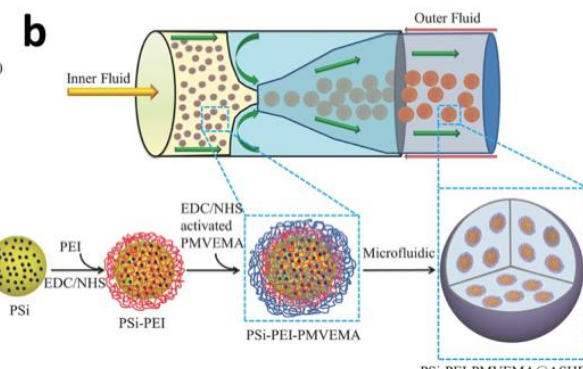

PSi-PEI-PMVEMA@ASH

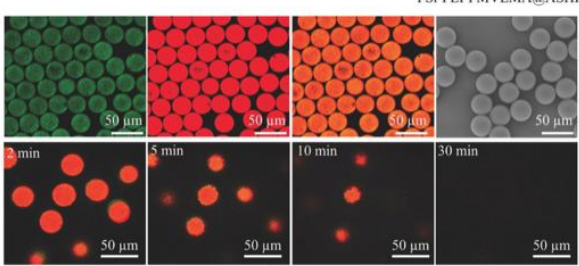

C

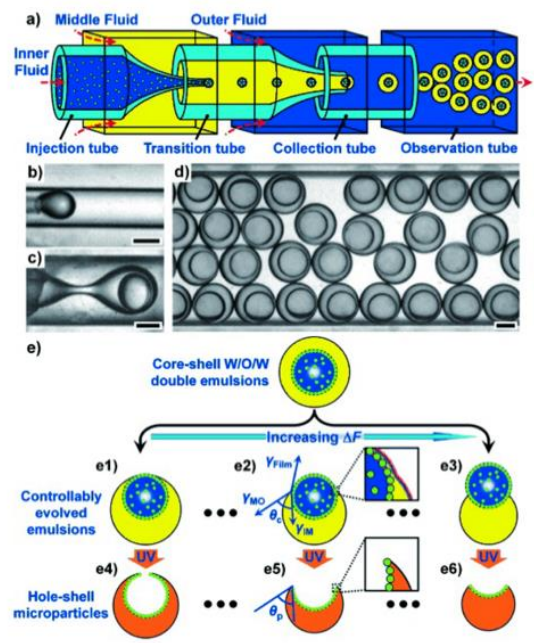

Figure 11. (a) Micrograph of solid polymeric particles derived from single emulsion droplets.

(b) Loading of nanoparticles within the polymeric matrix formed by single emulsion. A.

Schematic of the different stages of the production process; b. Confocal micrographs showing the homogeneity of the particles and the homogenous dispersion of the nanoparticles (FITC, green) within the polymeric matrix; c. Dissolution study of the microparticles over time at $\mathrm{pH}$ 7.4, illustrating the degradation profile of the system. (c) Core-shell particles obtained by double emulsion microfluidics. a. Schematic of the device engineered for the production of the double emulsions; $b-d$. Micrographs showing the formation of the inner droplets and the double emulsion; e. Schematic illustrating the template for the formation of the hole. Figures are reproduced with the permission from: (a) ref. ${ }^{[152]}$, Copyright 2009 , Wiley-VCH; (b) ref. ${ }^{[154]}$, Copyright 2014, WILEY-VCH. (c) ref. ${ }^{[156]}$, Copyright 2013, WILEY-VCH. 


\section{WILEY-VCH}

Emulsion droplets and microcapsules can serve also as microreactors, performing multiplex chemical or biological reactions on a small scale and with smaller consumption of reagents. ${ }^{[150 \text {, }}$

${ }^{157]}$ In particular, biological reactions, such as the widening of the polymerase reactions. ${ }^{[158]}$ The flow rate of both inner and outer phases have to be calibrated to ensure that each single droplet contained only one bacteria. After collecting the emulsions and performing the reaction, the droplets are run a second time through the chip, to create a water-in-oil-in-water $(\mathrm{w} / \mathrm{o} / \mathrm{w})$ emulsion easy to be read with instruments like flow cytometers (Figure 12a). ${ }^{[158]}$ Droplets microfluidics can also encapsulate cells for counting, further analysis, or for biomedical applications, with the caveat that, starting from a suspension of cells, their distribution in the droplets will follow a Poisson distribution. ${ }^{[7]}$ Thereby, on top of the control over the geometry of the device and the flow rates, a calculation and control over the concentration of the cells in the volume of the initial droplet is also required to avoid empty droplets. ${ }^{[150]}$ Each droplet can be barcoded to identify the droplet and its composition in the readout (Figure 12b). ${ }^{[159]}$ However, in particular applications, like the prolonged culture of cells structures, droplet emulsions are not considered the optimal mean, with hydrogel microcapsules representing the alternative (Figure 12c) ${ }^{[160]}$ Finally, multistep chemical and biological reactions can take place in single droplets through sequential injection of reagents (Figure 12d). 


\section{WILEY-VCH}

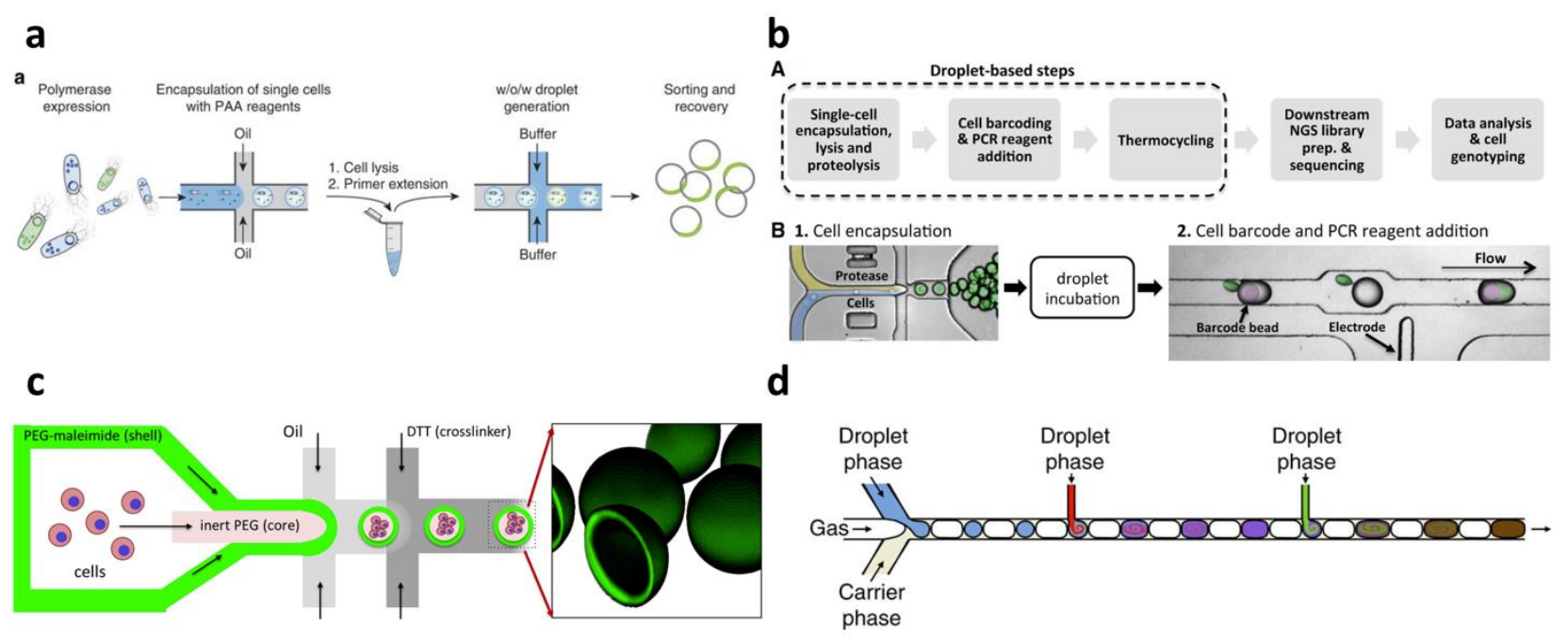

Figure 12. (a) Two-stage process for the improvement of polymerase chain reaction (PCR) reaction via droplet microfluidics. (b) Single-chip PCR reaction of encapsulated cells. a. Schematic of the process; b. Micrographs of the various on-chip steps. (c) On-chip cell encapsulation in hydrogels microcapsules. (d) Schematic of a multistep on-chip chemical reaction. Figures are reproduced with the permission from: (a) ref. ${ }^{[158]}$, Copyright 2016, the authors, under a Creative Common Attribution 4.0 International License. (b) ref. ${ }^{[161]}$, Copyright 2018, Pellegrino et al.; Published by Cold Spring Harbor Laboratory Press, under a Creative Common Attribution 4.0 International License. (c) ref. ${ }^{[160]}$, Copyright 2017 Acta Materialia Inc. Published by Elsevier Ltd. (d) ref. ${ }^{[162]}$, Copyright 2014, the authors, under a Creative Commons Attribution 3.0 Unported License.

\section{Conclusions and future perspectives}

Here, we have presented an overview of the basic mechanism and application of microfluidics for the production of both nano- and micro-particles or droplets with potential biomedical applications. Featured with identical properties, such as higher reproducibility, precisely controlled particle characteristics, tunable particle production efficient and feasible scale-up 


\section{WILEY-VCH}

processes, microfluidic platforms have unique advantages that may facilitate the clinical translation of cutting-edge technologies, including nanomedicines, single cell analysis and artificial cells. From an engineering point of view, for microfluidics-based nanoparticles fabrication, we have addressed the rationale of the advantageous features from microfluidic produced nanoparticles, and we highlighted the recent progresses of using microfluidics to synthesize nanoparticles with identical core/shell structures. Despite the prosper progress in core/shell nanoparticles fabrication and the following biomedical research, further work should be focused on unravel the mechanism of the core/shell structure formation, which may provide insights for guiding the nanosystem design. For microfluidics based micro-particle/droplets synthesis, we also illustrated the principle of design for microfluidic assisted micro-droplet production. In addition to their long established applications for drug encapsulation and delivery, we highlighted the integration of microfluidic produced micro-droplets in cellular analysis and simulation. We conclude that the future will see the mainstream application of droplet microfluidics to the high throughput screening of new drugs, and will allow the daily analysis of biological samples, as a result of the high reproducibility of the microfluidics technique.

\section{Acknowledgements}

Z. Liu acknowledges the financial support from Chinese Scholarship Council (CSC). F.F. acknowledges the Faculty of Pharmacy of the University of Helsinki for an assistant research grant. H.A.S. acknowledges financial support from the Sigrid Jusélius Foundation and the Helsinki Institute of Life Science 


\section{WILEY-VCH}

Received: ((will be filled in by the editorial staff))

Revised: ((will be filled in by the editorial staff))

Published online: ((will be filled in by the editorial staff))

\section{References}

[1] Z. Liu, V. Balasubramanian, C. Bhat, M. Vahermo, E. Mäkilä, M. Kemell, F. Fontana, A. Janoniene, V. Petrikaite, J. Salonen, Adv. Healthcare Mater. 2017, 6, 1601009; L. Wei, L. Yunzhan, L. Zehua, K. Nattha, Z. Ming, Z. Feng, L. Xueyan, B.-R. Tomás, L. Wenhua, Biomaterials 2018, 185, 322.

[2] Z. Wang, W. Liu, J. Shi, C. Nan, C. Fan, Mater. Horiz. 2018, 5, 344; F. Fontana, M. Fusciello, C. Groeneveldt, C. Capasso, J. Chiaro, S. Feola, Z. Liu, E. M. Mäkilä, J. J. Salonen, J. T. Hirvonen, ACS Nano 2019.

[3] Y. Ding, W. Li, F. Zhang, Z. Liu, N. Zanjanizadeh Ezazi, D. Liu, H. A. Santos, Adv. Funct. Mater. 2019, 29, 1802852; Y. Yu, G. Chen, J. Guo, Y. Liu, J. Ren, T. Kong, Y. Zhao, Mater. Horiz. 2018, 5, 1137.

[4] C. Xiong, J. Zhao, L. Wang, H. Geng, H. Xu, L. Yao, Mater. Horiz. 2017, 4, 862.

[5] A. Janoniene, Z. Liu, L. Baranauskiene, E. Mäkilä, M. Ma, J. Salonen, J. Hirvonen, H. Zhang, V. Petrikaite, H. A. Santos, ACS Appl. Mater. Interfaces 2017, 9, 13976; G. Bing, Z. Sheng, Kenry, D. Hu, X. Lin, S. Xu, C. Liu, H. Zheng, B. Liu, Mater. Horiz. 2017, 4, 1151.

[6] V. Balasubramanian, A. Correia, H. Zhang, F. Fontana, E. Mäkilä, J. Salonen, J. Hirvonen, H. A. Santos, Adv. Mater. 2017, 29, 1605375; D. Fan, E. Wang, S. Dong, Mater. Horiz. 2019, 6, 375.

[7] L. Mazutis, J. Gilbert, W. L. Ung, D. A. Weitz, A. D. Griffiths, J. A. Heyman, Nat. Protoc. 2013, 8, 870 . 


\section{WILEY-VCH}

[8] V. Balasubramanian, Z. Liu, J. Hirvonen, H. A. Santos, Adv. Healthcare Mater. 2018, 7, 1700432; M. Üner, S. A. Wissing, G. Yener, R. H. Müller, Adv. Drug Delivery Rev. 2005, 36, S131; L. F. Trierweiler, J. O. Trierweiler, Industrial Production of Polymeric Nanoparticles: Alternatives and Economic Analysis, 2011.

[9] R. Daw, J. Finkelstein, Nature 2006, 442, 367.

[10] D. Mark, S. Haeberle, G. Roth, F. V. Stetten, R. Zengerle, Chem. Soc. Rev. 2010, 39, 1153.

[11] G. M. Whitesides, Nature 2006, 442, 368; D. J. Beebe, G. A. M. And, G. M. Walker, Annu. Rev. Biomed. Eng. 2002, 4, 261; E. K. Sackmann, A. L. Fulton, D. J. Beebe, Nature 2014, 507, 181.

[12] P. M. Valencia, O. C. Farokhzad, K. Rohit, L. Robert, Nat. Nanotechnol. 2012, 7, 623.

[13] V. K. L. Mer, Ind. Eng. Chem. 1952, 44, 1270; V. K. Lamer, R. H. Dinegar, "Theory, Production and Mechanism of Formation of Monodispersed Hydrosols", presented at Hawaii International Conference on System Sciences, 1950.

[14] H. Reiss, J. Chem. Phys. 1951, 19, 482.

[15] I. M. Lifshitz, V. V. Slyozov, J. Phys. Chem. Solids 1961, 19, 35; C. Wagner, Elektrochemie 1961, 65, 581.

[16] P. W. Voorhees, J. Stat. Phys. 1985, 38, 231; Y. Liu, K. Kathan, W. Saad, R. K. Prud'homme, Phys. Rev. Lett. 2007, 98, 036102.

[17] N. T. K. Thanh, N. Maclean, S. Mahiddine, Chem. Rev. 2014, 114, 7610; T. Sugimoto, Adv. Colloid Interface Sci. 1987, 28, 65.

[18] K. Soon Gu, H. Taeghwan, Small 2011, 7, 2685.

[19] J. Polte, Cryst. Eng. Comm 2015, 17, 6809.

[20] B. K. Johnson, R. K. Prud'Homme, Phys. Rev. Lett. 2003, 91, 118302. 


\section{WILEY-VCH}

[21] S. M. D'Addio, R. K. Prud'Homme, Adv. Drug Delivery Rev. 2011, 63, 417.

[22] A. J. Mahajan, D. J. Kirwan, J. Cryst. Growth 1994, 144, 281.

[23] B. Bednár, K. Edwards, M. Almgren, S. Tormod, Z. Tuzar, Macromol. Rapid Commun. $1988,9,785$.

[24] O. Söhnel, J. W. Mullin, J. Colloid Interface Sci. 1988, 123, 43.

[25] S. D. Durbin, G. Feher, J. Cryst. Growth 1986, 76, 583.

[26] L. Dongfei, C. Salvatore, Z. Yuezhou, W. Chang-Fang, T. M. Sikanen, H. A. Santos, Adv. Mater. 2015, 27, 2269.

[27] W. S. Saad, R. K. Prud'Homme, Nano Today 2016, 11, 212; J. Andreas, S. M. Stavis, J. S. Hong, W. N. Vreeland, D. L. Devoe, G. Michael, ACS Nano 2010, 4, 2077.

[28] L. Jong-Min, S. Archana, L. M. Gilson, C. Sunandini, C. Sungyoung, W. Jun, L. Robert, K. Rohit, O. C. Farokhzad, ACS Nano 2014, 8, 6056.

[29] C. Y. Lee, C. L. Chang, Y. N. Wang, L. M. Fu, Int. J. Mol. Sci. 2011, 12, 3263.

[30] P. Jongnam, A. Kwangjin, H. Yosun, P. Je-Geun, N. Han-Jin, K. Jae-Young, P. JaeHoon, H. Nong-Moon, H. J. N. M. Taeghwan, Nat. Mater. 2004, 3, 891; Y. Song, J. Hormes, C. S. S. R. Kumar, Small 2010, 4, 698.

[31] T. J. Johnson, A. David Ross, L. E. Locascio, Anal. Chem. 2002, 74, 45.

[32] J. Atencia, D. J. Beebe, Nature 2005, 437, 648.

[33] J. D. Martin, S. D. Hudson, New J. Phys. 2009, 11, 26.

[34] in Encyclopedia of Microfluidics and Nanofluidics, (Ed: D. Li), Springer US, Boston, MA 2008, 1626.

[35] H. W. Prengle, G. Palm, in Thermodynamics of Solutions, Vol. 50, 2009, 848.

[36] in Encyclopedia of Microfluidics and Nanofluidics, (Ed: D. Li), Springer US, Boston, MA 2008, 1790. 


\section{WILEY-VCH}

[37] Y. Kim, C. B. Lee, M. Ma, W. J. Mulder, Z. A. Fayad, O. C. Farokhzad, R. Langer, Nano Lett. 2013, 13, 4997.

[38] B. D. Guy, C. Jacob, Phys. Rev. Lett. 2007, 98, 064503.

[39] P. Gravesen, J. Branebjerg, O. S. Jensen, J. Micromech. Microeng. 1993, 3, 168.

[40] X. F. Peng, G. P. Peterson, B. X. Wang, Fuel Energy Abstr. 1994, 7, 249.

[41] M. Mala, L. I. Dongqing, Int. J. Heat Fluid Flow 1999, 20, 142.

[42] G. R. Wang, F. Yang, W. Zhao, Lab Chip 2014, 14, 1452; T. Burghelea, E. Segre, V. Steinberg, Phys. Rev. Lett. 2004, 92, 164501.

[43] R. Othman, G. T. Vladisavljević, H. C. H. Bandulasena, Z. K. Nagy, Chem. Eng. J. 2015, $280,316$.

[44] D. Liu, H. Zhang, S. Cito, J. Fan, E. Mäkilä, J. Salonen, J. Hirvonen, T. M. Sikanen, D. A. Weitz, H. A. Santos, Nano Lett. 2017, 17, 606.

[45] D. E. Hershey, B. G. Thomas, F. M. Najjar, Int. J. Numer. Methods Fluids 2010, 17, 23.

[46] C. C. Hong, J. W. Choi, C. H. Ahn, Lab Chip 2004, 4, 109; P. M. Valencia, P. A. Basto, Z. Liangfang, R. Minsoung, L. Robert, O. C. Farokhzad, K. Rohit, ACS Nano 2010, 4, 1671.

[47] H. Kim, J. Sung, Y. Chang, A. Alfeche, C. Leal, ACS Nano, 12, 9196.

[48] A. D. Stroock, S. K. Dertinger, A. Ajdari, I. Mezic, H. A. Stone, G. M. Whitesides, Science 2002, 295, 647.

[49] A. J. Demello, Nature 2006, 442, 394; K. Y. Tung, C. C. Li, J. T. Yang, Microfluid. Nanofluid. 2009, 7, 545.

[50] J. Li, G. Xia, Y. Li, J. Chem. Technol. Biotechnol. 2013, 88, 1757.

[51] J. Sun, Y. Xianyu, M. Li, W. Liu, L. Zhang, D. Liu, C. Liu, G. Hu, X. Jiang, Nanoscale $2013,5,5262$. 


\section{WILEY-VCH}

[52] S. Jiashu, Z. Lu, W. Jiuling, F. Qiang, L. Dingbin, Y. Qifang, X. Dongyan, W. Yujie, D. Baoquan, S. Xinghua, Adv. Mater. 2015, 27, 1402.

[53] R. Donno, A. Gennari, E. Lallana, D. L. R. Jmr, R. D'Arcy, K. Treacher, K. Hill, M. Ashford, N. Tirelli, Int. J. Pharm. 2017, 534, 97; Q. Nan, B. Li, H. You, Z. Wei, L. Fu, Y. Wang, L. Chen, Anal. Methods 2014, 6, 4077; R. D. Santo, L. Digiacomo, S. Palchetti, V. Palmieri, G. Caracciolo, Nanoscale 2019, 11, 2733.

[54] N. Kimura, M. Maeki, Y. Sato, Y. Note, A. Ishida, H. Tani, H. Harashima, M. Tokeshi, ACS Omega 2018, 3, 5044.

[55] A. Bokare, A. Takami, J. H. Kim, A. Dong, A. Chen, R. Valerio, S. Gunn, F. Erogbogbo, ACS Omega 2019, 4, 4650.

[56] A. Bains, Y. Cao, M. G. Moffitt, Macromol. Rapid Commun. 2016, 36, 2000; S. Greg, W. Chih-Wei, O. Ali, Y. Huda, M. G. Moffitt, S. David, Langmuir 2008, 24, 10596; W. ChihWei, O. Ali, S. David, M. G. Moffitt, Langmuir 2010, 26, 716; M. Lopez, M. D. Graham, Phys. Fluids 2008, 20, 2376.

[57] Z. Xu, C. Lu, J. Riordon, D. Sinton, M. G. Moffitt, Langmuir 2016, 32, 12781.

[58] R. Mukhopadhyay, Anal. Chem. 2007, 79, 3248.

[59] R. Kangning, Z. Jianhua, W. Hongkai, Acc. Chem. Res. 2013, 46, 2396; X. Mu, Q. Liang, P. Hu, K. Ren, Y. Wang, G. Luo, Lab Chip 2009, 9, 1994.

[60] W. Wang, S. Zhao, T. Shao, Y. Jin, Y. Cheng, Chem. Eng. J. 2012, 192, 252.

[61] A.-S. Yang, F.-C. Chuang, C.-K. Chen, M.-H. Lee, S.-W. Chen, T.-L. Su, Y.-C. Yang, Chem. Eng. J. 2015, 263, 444.

[62] N. Hao, Y. Nie, Z. Xu, J. X. J. Zhang, J. Colloid Interface Sci. 2019, 542, 370.

[63] M. Wiese, S. Benders, B. Blümich, M. Wessling, Chem. Eng. J. 2018, 343, 54.

[64] M. K. Parsa, F. Hormozi, D. Jafari, Comput. Fluids 2014, 105, 82. 


\section{WILEY-VCH}

[65] Z. Yang, S. Matsumoto, H. Goto, M. Matsumoto, R. Maeda, Sens. Actuators, A 2001, 93, 266.

[66] H. Song, R. F. Ismagilov, J. Am. Chem. Soc. 2003, 125, 14613; G. G. Yaralioglu, I. O. Wygant, T. C. Marentis, B. T. Khuri-Yakub, Anal. Chem. 2004, 76, 3694.

[67] D. Ahmed, X. Mao, B. K. Juluri, T. J. Huang, Microfluid. Nanofluid. 2009, 7, 727.

[68] K. Sritharan, C. J. Strobl, M. F. Schneider, A. Wixforth, Z. v. Guttenberg, Appl. Phys. Lett. 2006, 88, 054102; Q. Zeng, F. Guo, L. Yao, H. W. Zhu, L. Zheng, Z. X. Guo, W. Liu, Y. Chen, S. S. Guo, X. Z. Zhao, Sens. Actuators, B 2011, 160, 1552.

[69] G. Luo, L. Du, Y. Wang, K. Wang, Chem. Eng. Technol., 42, 1; E. Swider, O. Koshkina, J. Tel, L. J. Cruz, I. J. M. de Vries, M. Srinivas, Acta Biomater. 2018, 73, 38.

[70] J. Ahn, J. Ko, S. Lee, J. Yu, Y. Kim, N. L. Jeon, Adv. Drug Delivery Rev. 2018, 128, 29; S. Colombo, M. Beck-Broichsitter, J. P. Bøtker, M. Malmsten, J. Rantanen, A. Bohr, Adv. Drug Delivery Rev. 2018, 128, 115.

[71] S. T. Sanjay, W. Zhou, M. Dou, H. Tavakoli, L. Ma, F. Xu, X. Li, Adv. Drug Delivery Rev. 2018, 128, 3; D. Liu, H. Zhang, F. Fontana, J. T. Hirvonen, H. A. Santos, Adv. Drug Delivery Rev. 2018, 128, 54.

[72] M. Brzeziński, M. Socka, B. Kost, Polym. Int. 2019, 68, 997.

[73] C. R. Ghosh, S. J. C. R. Paria, Chem. Rev. 2012, 112, 2373.

[74] S. Ding, F. A. Mohamed, J. Wallyn, C. Taddei, V. Thierry, Langmuir 2018, 34, 1981.

[75] D. Liu, C. R. Bernuz, J. Fan, W. Li, A. Correia, J. Hirvonen, H. A. Santos, Adv. Funct. Mater. 2017, 27, 1604508.

[76] P. M. Valencia, P. A. Basto, L. Zhang, M. Rhee, R. Langer, O. C. Farokhzad, R. Karnik, ACS nano 2010, 4, 1671. 


\section{WILEY-VCH}

[77] L. Rao, B. Cai, L. L. Bu, Q. Q. Liao, S. S. Guo, X. Z. Zhao, W. F. Dong, W. Liu, ACS Nano 2017, 11, 3496.

[78] F. C. Cabrera, A. F. A. A. Melo, J. O. C. P. D. Souza, A. E. Job, F. N. Crespilho, Lab Chip 2015, 15, 1835.

[79] H. S. Mohammad Mahdi, D. Erfan, B. Ghasem, F. S. Majedi, K. Hamid, J. J. V. Dersarl, B. Arnaud, P. Arash, R. Philippe, T. Lobat, Nanomedicine 2015, 10, nnm.15.162.

[80] Z. Liu, Y. Li, W. Li, C. Xiao, D. Liu, C. Dong, M. Zhang, E. MãaKilãa, M. Kemell, J. Salonen, Adv. Mater. 2017, 30, 1703393.

[81] Z. Liu, Y. Li, W. Li, W. Lian, M. Kemell, S. Hietala, P. Figueiredo, L. Li, E. Mäkilä, M. Ma, Mater. Horiz. 2019, 6, 385.

[82] D. Liu, H. Zhang, E. Mäkilä, J. Fan, B. Herranz-Blanco, C. F. Wang, R. Rosa, A. J. Ribeiro, J. Salonen, J. Hirvonen, Biomaterials 2015, 39, 249.

[83] H. Zhang, D. Liu, L. Wang, Z. Liu, R. Wu, A. Janoniene, M. Ma, G. Pan, L. Baranauskiene, L. Zhang, Adv. Healthcare Mater. 2017, 6, 1601406.

[84] W. Lei, S. Ma, B. Yang, W. Cao, X. Han, Chem. Eng. J. 2015, 268, 102.

[85] S. Y. Tang, R. Qiao, S. Yan, D. Yuan, Q. Zhao, G. Yun, T. P. Davis, W. Li, Small 2018, $14,1800118$.

[86] J. o. P. Martins, D. Liu, F. Fontana, M. P. A. Ferreira, A. Correia, S. Valentino, M. Kemell, K. Moslova, E. Mäkilä, J. Salonen, ACS Appl. Mater. Interfaces 2018, 10, 44354.

[87] J. A. Kulkarni, T. Yyc, S. Chen, Y. K. Tam, J. Zaifman, P. R. Cullis, S. Biswas, Nanoscale 2017, 9, 13600.

[88] R. Di Santo, L. Digiacomo, S. Palchetti, V. Palmieri, G. Perini, D. Pozzi, M. Papi, G. Caracciolo, Nanoscale 2019, 11, 2733. 


\section{WILEY-VCH}

[89] L. Gomez, V. Sebastian, S. Irusta, A. Ibarra, M. Arruebo, J. Santamaria, Lab Chip 2013, 14,325 .

[90] K. Shiba, T. Sugiyama, T. Takei, G. Yoshikawa, Chem. Commun. 2015, 51, 15854.

[91] Z. Lu, F. Qiang, W. Jiuling, Z. Shuai, D. Baoquan, W. Yujie, D. Mingdong, R. Ji-Young, Y. Tae-Young, S. Xinghua, ACS Nano 2015, 9, 9912.

[92] Z. Lu, F. Qiang, W. Jiuling, S. Jiashu, S. Xinghua, J. Xingyu, Angew. Chem., Int. Ed. $2015,54,3830$

[93] S. Tao, M. Yang, H. Chen, M. Ren, G. Chen, J. Colloid Interface Sci. 2016, 486, 16.

[94] M. M. Hasani-Sadrabadi, S. Taranejoo, E. Dashtimoghadam, G. Bahlakeh, F. S. Majedi, J. J. Vandersarl, M. Janmaleki, F. Sharifi, A. Bertsch, K. Hourigan, Adv. Mater. 2016, 28, 4134.

[95] R. H. Fang, A. V. Kroll, W. Gao, L. Zhang, Adv. Mater. 2018, 30, 1706759.

[96] A. M. Derfus, W. C. W. Chan, S. N. Bhatia, Adv. Mater. 2010, $16,961$.

[97] M. E. Gindy, A. Z. Panagiotopoulos, R. K. Prud'Homme, Langmuir 2008, 24, 83.

[98] R. F. Pagels, J. Edelstein, C. Tang, R. K. Prud'Homme, Nano Lett. 2018, 18, 1139.

[99] N. M. Pinkerton, M. E. Gindy, V. L. Calero-Ddelc, T. Wolfson, R. F. Pagels, D. Adler, D. Gao, S. Li, R. Wang, M. Zevon, Adv. Healthcare Mater. 2015, 4, 1376.

[100] K. Meleson, S. Graves, T. G. Mason, Soft Mater. 2004, 2, 109; S. Ding, M. F. Attia, J. Wallyn, C. Taddei, C. A. Serra, N. Anton, M. Kassem, M. Schmutz, M. Er-Rafik, N. Messaddeq, Langmuir 2018, 34, 1981.

[101] A. Gupta, H. B. Eral, T. A. Hatton, P. S. Doyle, Soft Matter 2016, 12, 1452.

[102] N. J. Carroll, S. Pylypenko, P. B. Atanassov, D. N. Petsev, Langmuir 2009, 25, 13540;

C. H. Yang, Y. S. Lin, K. S. Huang, Y. C. Huang, E. C. Wang, J. Y. Jhong, C. Y. Kuo, Lab Chip 2009, 9, 145; H. C. Shum, J. W. Kim, D. A. Weitz, J. Am. Chem. Soc. 2008, 130, 9543. [103] D. Liu, H. Zhang, F. Fontana, J. T. Hirvonen, H. A. Santos, Lab Chip 2017, 17, 1856. 


\section{WILEY-VCH}

[104] F. Fontana, M. P. A. Ferreira, A. Correia, J. Hirvonen, H. A. Santos, J. Drug Delivery Sci. Technol. 2016, 34, 76.

[105] W. J. Duncanson, T. Lin, A. R. Abate, S. Seiffert, R. K. Shah, D. A. Weitz, Lab Chip 2012, 12, 2135; S. Seiffert, Angew. Chem., Int. Ed. Engl. 2013, 52, 11462.

[106] D. J. McClements, Curr. Opin. Colloid Interface Sci. 2012, 17, 235.

[107] T. Tadros, P. Izquierdo, J. Esquena, C. Solans, Adv. Colloid. Interface. Sci. 2004, 108109, 303.

[108] T. S. H. Leong, T. J. Wooster, S. E. Kentish, M. Ashokkumar, Ultrason. Sonochem. $2009,16,721$.

[109] F. Macritchie, Nature 1967, 215, 1159.

[110] B. E. Rapp, Microfluidics: Modeling, Mechanics and Mathematics, William Andrew, 2016.

[111] L. Menetrier-Deremble, P. Tabeling, Phys. Rev. E 2006, 74, 035303.

[112] P. Garstecki, M. J. Fuerstman, H. A. Stone, G. M. Whitesides, Lab Chip 2006, 6, 437.

[113] D. R. Link, S. L. Anna, D. A. Weitz, H. A. Stone, Phys. Rev. Lett. 2004, 92, 054503.

[114] P. Guillot, A. Colin, Phys. Rev. E 2005, 72, 066301.

[115] A. S. Utada, A. Fernandez-Nieves, J. M. Gordillo, D. A. Weitz, Phys. Rev. Lett. 2008, 100,014502

[116] W. Li, L. Zhang, X. Ge, B. Xu, W. Zhang, L. Qu, C.-H. Choi, J. Xu, A. Zhang, H. Lee, Chem. Soc. Rev. 2018, 47, 5646; J. Koh, C.-Y. Wu, H. Kittur, D. Di Carlo, Lab Chip 2015, 15, 3818; S. Ding, C. A. Serra, T. F. Vandamme, W. Yu, N. Anton, J. Controlled Release 2018, 295, 31; F. He, M. J. Zhang, W. Wang, Q. W. Cai, Y. Y. Su, Z. Liu, Y. Faraj, X. J. Ju, R. Xie, L. Y. Chu, Adv. Mater. Technol. 2019, 1800687. 


\section{WILEY-VCH}

[117] L. Y. Chu, A. S. Utada, R. K. Shah, J. W. Kim, D. A. Weitz, Angew. Chem., Int. Ed. Engl. 2007, 46, 8970.

[118] B. Herranz-Blanco, E. Ginestar, H. Zhang, J. Hirvonen, H. A. Santos, Int. J. Pharm. $2017,516,100$.

[119] S. Seiffert, J. Thiele, A. R. Abate, D. A. Weitz, J. Am. Chem. Soc. 2010, 132, 6606.

[120] S. L. Anna, N. Bontoux, H. A. Stone, Appl. Phys. Lett. 2003, 82, 364.

[121] K. L. Lao, J. H. Wang, G. B. Lee, Microfluid. Nanofluid. 2009, 7, 709.

[122] R. K. Shah, H. C. Shum, A. C. Rowat, D. Lee, J. J. Agresti, A. S. Utada, L. Y. Chu, J. W. Kim, A. Fernandez-Nieves, C. J. Martinez, D. A. Weitz, Mater. Today 2008, 11, 18.

[123] V. Balasubramanian, B. Herranz-Blanco, P. V. Almeida, J. Hirvonen, H. A. Santos, Prog. Polym. Sci. 2016, 60, 51.

[124] M. Brzeziński, Macromol. Chem. Phys. 2017, 218, 1700018.

[125] E. Lorenceau, A. S. Utada, D. R. Link, G. Cristobal, M. Joanicot, D. A. Weitz, Langmuir $2005,21,9183$.

[126] H. C. Shum, D. Lee, I. Yoon, T. Kodger, D. A. Weitz, Langmuir 2008, 24, 7651.

[127] S. Ota, S. Yoshizawa, S. Takeuchi, Angew. Chem., Int. Ed. Engl. 2009, 48, 6533.

[128] S. H. Kim, J. W. Kim, D. H. Kim, S. H. Han, D. A. Weitz, Microfluid. Nanofluid. 2013, $14,509$.

[129] R. C. Hayward, A. S. Utada, N. Dan, D. A. Weitz, Langmuir 2006, 22, 4457.

[130] F. Tu, D. Lee, Langmuir 2012, 28, 9944.

[131] L. R. Arriaga, S. S. Datta, S. H. Kim, E. Amstad, T. E. Kodger, F. Monroy, D. A. Weitz, Small 2014, 10, 950.

[132] T. Robinson, Adv Biosyst 2019, 3.

[133] S. Matosevic, Bioessays 2012, 34, 992. 


\section{WILEY-VCH}

[134] A. Armada-Moreira, E. Taipaleenmaki, F. Itel, Y. Zhang, B. Stadler, Nanoscale 2016, $8,19510$.

[135] S. Deshpande, C. Dekker, Nat. Protoc. 2018, 13, 856.

[136] D. van Swaay, A. deMello, Lab Chip 2013, 13, 752.

[137] M. Weiss, J. P. Frohnmayer, L. T. Benk, B. Haller, J. W. Janiesch, T. Heitkamp, M.

Borsch, R. B. Lira, R. Dimova, R. Lipowsky, E. Bodenschatz, J. C. Baret, T. Vidakovic-Koch, K. Sundmacher, I. Platzman, J. P. Spatz, Nat. Mater. 2018, 17, 89.

[138] P. Schwille, J. Spatz, K. Landfester, E. Bodenschatz, S. Herminghaus, V. Sourjik, T. J. Erb, P. Bastiaens, R. Lipowsky, A. Hyman, P. Dabrock, J. C. Baret, T. Vidakovic-Koch, P. Bieling, R. Dimova, H. Mutschler, T. Robinson, T. D. Tang, S. Wegner, K. Sundmacher, Angew Chem Int Ed Engl 2018, 57, 13382.

[139] D. Merkle, N. Kahya, P. Schwille, Chembiochem 2008, 9, 2673; C. Campillo, B. PepinDonat, A. Viallat, Soft Matter 2007, 3, 1421; C. Martino, T. Y. Lee, S. H. Kim, A. J. deMello, Biomicrofluidics 2015, 9, 024101.

[140] C. Martino, C. Statzer, D. Vigolo, A. J. deMello, Lab Chip 2016, 16, 59.

[141] S. H. Kim, J. W. Kim, D. H. Kim, S. H. Han, D. A. Weitz, Small 2013, 9, 124.

[142] T. Sanchez, D. T. Chen, S. J. DeCamp, M. Heymann, Z. Dogic, Nature 2012, 491, 431.

[143] C. Martino, A. J. deMello, Interface Focus 2016, 6, 20160011.

[144] F. Fanalista, A. Birnie, R. Maan, F. Burla, K. Charles, G. Pawlik, S. Deshpande, G. H. Koenderink, M. Dogterom, C. Dekker, ACS Nano 2019, 13, 5439.

[145] R. J. Peters, M. Marguet, S. Marais, M. W. Fraaije, J. C. van Hest, S. Lecommandoux, Angew. Chem., Int. Ed. Engl. 2014, 53, 146.

[146] N. N. Deng, M. Yelleswarapu, L. F. Zheng, W. T. S. Huck, J. Am. Chem. Soc. 2017, $139,587$. 


\section{WILEY-VCH}

[147] K. Gopfrich, I. Platzman, J. P. Spatz, Trends Biotechnol 2018, 36, 938.

[148] M. Ugrinic, A. Zambrano, S. Berger, S. Mann, T. D. Tang, A. deMello, Chem. Commun. 2018, 54, 287.

[149] Y. Elani, T. Trantidou, D. Wylie, L. Dekker, K. Polizzi, R. V. Law, O. Ces, Sci. Rep. $2018,8,4564$.

[150] S. Mashaghi, A. Abbaspourrad, D. A. Weitz, A. M. van Oijen, Trac-Trends Anal. Chem. $2016,82,118$.

[151] W. Wang, M. J. Zhang, L. Y. Chu, Acc Chem Res 2014, 47, 373.

[152] Q. Xu, M. Hashimoto, T. T. Dang, T. Hoare, D. S. Kohane, G. M. Whitesides, R. Langer, D. G. Anderson, Small 2009, 5, 1575.

[153] D. Liu, B. Herranz-Blanco, E. Makila, L. R. Arriaga, S. Mirza, D. A. Weitz, N. Sandler, J. Salonen, J. Hirvonen, H. A. Santos, ACS Appl. Mater. Interfaces 2013, 5, 12127.

[154] H. Zhang, D. Liu, M. A. Shahbazi, E. Makila, B. Herranz-Blanco, J. Salonen, J. Hirvonen, H. A. Santos, Adv. Mater. 2014, 26, 4497.

[155] J. Wu, T. Kong, K. W. Yeung, H. C. Shum, K. M. Cheung, L. Wang, M. K. To, Acta Biomater. 2013, 9, 7410; M. Brzeziński, A. Kacprzak, M. Calderón, S. Seiffert, Macromol. Rapid. Commun. 2017, 38, 1600790.

[156] W. Wang, M. J. Zhang, R. Xie, X. J. Ju, C. Yang, C. L. Mou, D. A. Weitz, L. Y. Chu, Angew. Chem., Int. Ed. Engl. 2013, 52, 8084.

[157] H. Song, D. L. Chen, R. F. Ismagilov, Angew. Chem., Int. Ed. Engl. 2006, 45, 7336; L. Shang, Y. Cheng, Y. Zhao, Chem. Rev. 2017, 117, 7964; H. Tanaka, S. Yamamoto, A. Nakamura, Y. Nakashoji, N. Okura, N. Nakamoto, K. Tsukagoshi, M. Hashimoto, Anal. Chem. 2015, 87, 4134; Y. Ding, J. Choo, A. J. deMello, Microfluid. Nanofluid. 2017, 21. 


\section{WILEY-VCH}

[158] A. C. Larsen, M. R. Dunn, A. Hatch, S. P. Sau, C. Youngbull, J. C. Chaput, Nat. Commun. 2016, 7, 11235.

[159] E. Brouzes, M. Medkova, N. Savenelli, D. Marran, M. Twardowski, J. B. Hutchison, J. M. Rothberg, D. R. Link, N. Perrimon, M. L. Samuels, Proc. Natl. Acad. Sci. U. S. A. 2009, $106,14195$.

[160] C. Siltanen, M. Diakatou, J. Lowen, A. Haque, A. Rahimian, G. Stybayeva, A. Revzin, Acta Biomater. 2017, 50, 428.

[161] M. Pellegrino, A. Sciambi, S. Treusch, R. Durruthy-Durruthy, K. Gokhale, J. Jacob, T. X. Chen, J. A. Geis, W. Oldham, J. Matthews, H. Kantarjian, P. A. Futreal, K. Patel, K. W. Jones, K. Takahashi, D. J. Eastburn, Genome. Res. 2018, 28, 1345.

[162] A. M. Nightingale, T. W. Phillips, J. H. Bannock, J. C. de Mello, Nat. Commun. 2014, 5,3777 . 


\section{WILEY-VCH}

\section{Microfluidics for production of particles: mechanism, methodology and applications}

Keyword microfluidics; nanoparticles; microparticles; drug delivery; biological analysis

Z. Liu, F. Fontana, A. Python, J. T. Hirvonen, and H. A. Santos*

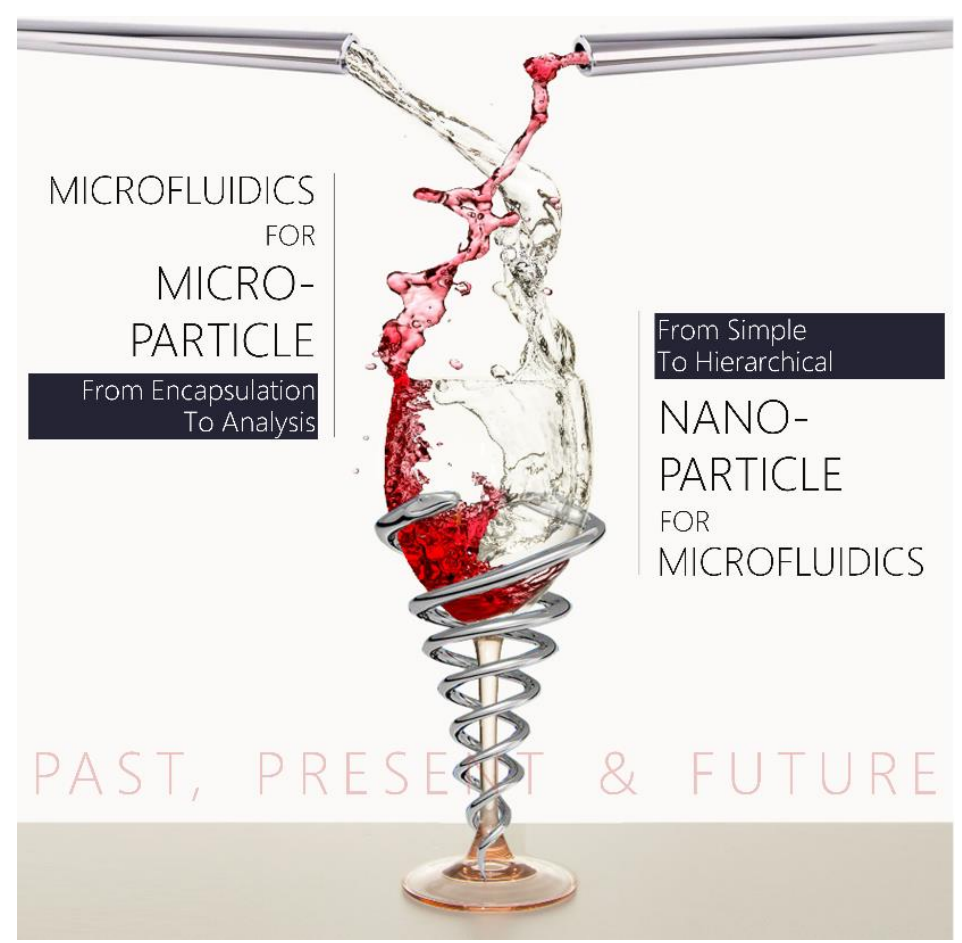

As a fundamental compartment, micro- and nano-particles or droplets have been extensively applied for biomedical usages. A review of microfluidic produced nano-, micro-particles or droplets and a brief summary of their corresponding mechanism and production is presented. Previous mechanical theories are explained, current tendency of their actual application is discussed and the future perspectives and limitations are proposed. 